\title{
Strong Self-Propelled Swing Motion Due to the Growing Instability on Heat Transfer
}

\author{
Hideyuki Sugioka* and Mako Kubota \\ Department of Mechanical Systems Engineering, \\ Shinshu University 4-17-1 Wakasato, Nagano 380-8553, Japan
}

(Dated: April 21, 2020)

\begin{abstract}
We report that pendulums having a hot tip immersed in water exhibit a strong self-propelled swing motion. This large swing motion is observed when the temperature of the hot tip becomes that of the nucleate- to film-boiling regime. We propose a model that explains how the strong swing motion occurs owing to the growing asymmetrical instability of heat transfer from the hot tip to the water; i.e., the small swing motion increases the asymmetrical heat transfer and consequently accelerates the swing motion in the liquid. We believe that our findings will contribute significantly to a new class of self-propelled devices and energy conversion systems that convert waste heat into usable energy.
\end{abstract}

PACS numbers: 47.85.L-s, 47.55.D-, 68.03.-g

\footnotetext{
* hsugioka@shinshu-u.ac.jp
} 


\section{INTRODUCTION}

Heat engines are machines that can convert heat energy into mechanical energy, and they are a key technology for sustaining the modern industrialized society. However, heat engines typically possess an expensive complex structure. Therefore, heat engines with a simple structure are attractive as a new class of engine, particularly from the perspective of low energy harvesting, as a large amount of heat energy is lost as waste heat. In this context, Linke et al. [1] reported that liquids exhibit self-propelled motion at speeds of $\sim 5$ $\mathrm{cm} / \mathrm{s}$ due to Leidenfrost phenomena $[2,3]$ when they are placed in contact with hot surfaces having an asymmetrical ratchetlike topology. They suggested that this occurrence would aid the construction of pumps that are powered by waste heat. Shortly afterwards, Ok et al. [4] demonstrated that sub-micron ratchets yield water droplet velocities reaching $\sim 40 \mathrm{~cm} / \mathrm{s}$. Li et al. [5] presented a lattice Boltzmann modeling of the self-propelled Leidenfrost droplets, whereas Würger [6] proposed a thermal creep model. Hashmi et al. [7] demonstrated that a small cart can be levitated by its own vapor layer and can move without friction over the hot ratchet. Furthermore, by extending the concept of Linke et al.'s device, Wells et al. [8] demonstrated that dry-ice blocks rotate on hot turbine-like surfaces and thus can covert the latent heat into rotational motion. Moreover, from the viewpoint of new micro heat engines and these applications, many challenging studies have been done recently. For example, Shi et al. [9] showed that a micro-hovercraft can move at a speed of $\sim 7 \mathrm{~cm} / \mathrm{s}$ by using the cold Leidenfrost phenomenon. Sugioka and Segawa [10] demonstrated that a micro-glider having a ratchet structure moves on a shallow water layer at a speed of $\sim 20$ $\mathrm{cm} / \mathrm{s}$. Xu et al. [11] presented self-propelled rotational Leidenfrost rotor made of wet filter paper that exhibits angular velocities exceeding $30 \mathrm{rad} / \mathrm{s}$. In addition, Luo et al. [12] showed the self-propulsion of Leidenfrost drops between non-parallel structures. Pham et al. [13] demonstrated that hydrogel drops initially at rest on a surface spontaneously jump upon rapid heating and continue to bounce with increasing amplitudes. Bouillant et al. [14] reported that Leidenfrost droplets initially at rest on horizontal substrates self-rotate and self-propel in the direction that they are rolling.

However, the self-propelled devices using ordinary Leidenfrost phenomena require an extremely large heat flux exceeding approximately $1 \mathrm{GW} / \mathrm{m}^{2}$, whereas the self-propelled devices using cold Leidenfrost phenomena need an extremely cold working medium (e.g., 
dry-ice) to sublimate. For example, if we attempt to realize an auto cooling pump (or a power generation system on the roof) by using Linke et al.'s device [1], water is dried immediately before the Linke et al.'s device starts to work. This is because, generally, we cannot place liquids suddenly in contact with a hot plate at a temperature exceeding

the Leidenfrost temperature without using special electronic devices that waste energy [2]. Therefore, to overcome this problem, we must find a self-propelled device that functions at lower temperatures (e.g., near the boiling temperature or the nucleate-boiling regime) and at lower heat fluxes. Nevertheless, a self-propelled device using a heat energy in the nucleateto film-boiling regime has not been explored well to the best our knowledge, although the heat transfer phenomena in the nucleate- to film-boiling regime have been studied well [15].

Here, we report that pendulums having a hot tip partially immersed in water, exhibit a strong self-propelled swing motion with a tip velocity on the order of $6 \mathrm{~cm} / \mathrm{s}$ in the nucleateto film-boiling regime at a heat flux $q \sim 5 \mathrm{MW} / \mathrm{m}^{2}$, which is 200 times smaller than that of Linke et al.'s device. Thus, in contrast to Linke et al.'s device, the self-swing motion in our study can be realized even if the tip temperature increases gradually. Therefore, it is applicable to innovative energy harvesting devices. Further, because the tip velocity of $\sim 6$ $\mathrm{cm} / \mathrm{s}$ is a remarkable speed for an object in water using a device with a simple structure, it is applicable to innovative microfluidic devices. Furthermore, we propose a model that explains how the strong swing motion occurs owing to the growing asymmetrical instability of heat transfer from the hot tip to the water; i.e., the small swing motion increases the asymmetrical heat transfer and consequently accelerates the swing motion in the liquid.

\section{EXPERIMENTAL METHOD}

Figure 1 shows a schematic of the experimental setup for the self-propelled swing motion. As shown in Fig. 1, we prepared a pendulum having a heater made of a U-shaped nichrome wire of diameter $\phi_{1}=0.26 \mathrm{~mm}$, real length $l_{h}=16$ to $20 \mathrm{~mm}$, and surface area $A=\pi \phi_{1} l_{h}=$ 13.1 to $16.3 \mathrm{~mm}^{2}$. Here, the other part of the pendulum was made of two copper wires of diameter $\phi_{2}=0.9 \mathrm{~mm}$. Typically, the total length $L$ of the pendulum is 100 to $150 \mathrm{~mm}$ and the projection length $L_{h}$ of the U-shaped heater 6 to $9 \mathrm{~mm}$. Furthermore, we immersed a region of length $L_{w}$ (typically, 9 to $12 \mathrm{~mm}$ ) from the tip in deionized water (milli-Q, 18.2 $\mathrm{M} \Omega \mathrm{cm}$ ) at rest. Then, upon applying a DC electric voltage $V_{0}$ to the nichrome wire (with 


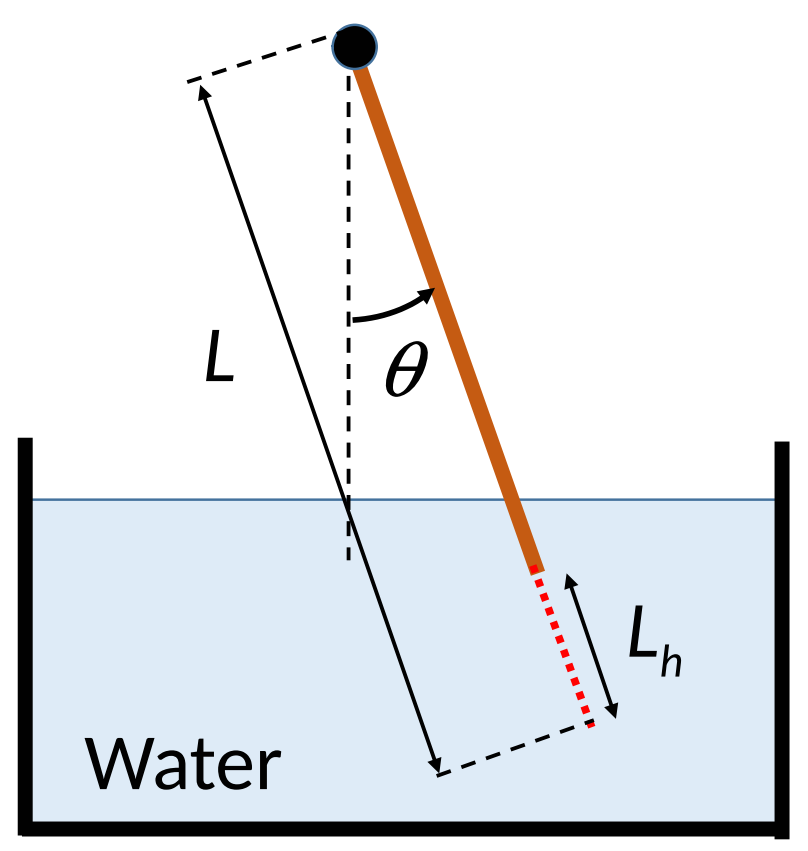

FIG. 1. (Color online) Experimental setup for the self-propelled swing motion. Here, typically, $L=100$ to $150 \mathrm{~mm}, L_{h}=6$ to $9 \mathrm{~mm}$, and $\Theta=0 \mathrm{rad}$.

a current $I_{0}$ and a power $P_{0}$ ), we observed a significant self-propelled swing motion with a sound. Subsequently, we determined the displacement $X_{p}$ of the tip of the pendulum in the $x$ direction at time $t$ by using video data of size $640 \times 480$ with a frame rate of $240 \mathrm{fps}$. Such measurements were repeated $N_{f}$ times (typically, $N_{f}=3$ ) at $P_{0}$. Note that, in Fig. $1, \Theta$ is the bending angle of the heater. However, as shown in Fig. 4(b), because the swing motion did not depend significantly on $\Theta$, we set $\Theta=0$ rad for almost all experiments.

\section{EXPERIMENTAL RESULTS}

Figure 2 shows the photographs of the observed typical self-propelled motion at $t=0$ to $18.38 \mathrm{~s}$ when $V_{0}=7.7 \mathrm{~V}, I_{0}=9.5 \mathrm{~A}, P_{0}=73.2 \mathrm{~W}, R_{0}=0.81 \Omega, L=150 \mathrm{~mm}, L_{h}=9$ $\mathrm{mm}, l_{h}=20 \mathrm{~mm}, \phi_{1}=0.26 \mathrm{~mm}, A=\pi \phi_{1} l_{h}=16.3 \mathrm{~mm}^{2}$, and $L_{w}=12 \mathrm{~mm}$. In Fig. 2(a), we evidence bubbles by using a dark background and front illumination. Thus, the white clouds in Figs. 2(b) and 2(c) show the bubble cloud that is released from the heater. As shown in Figs. 2(a) to 2(c), we find that the swing motion does not occur and the bubble 


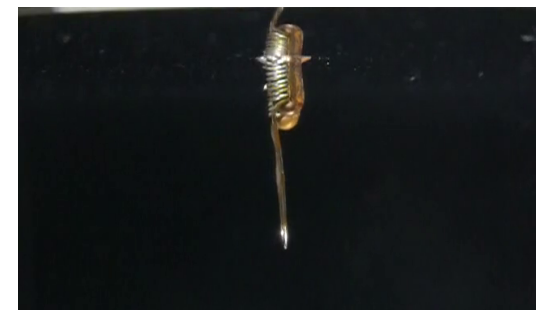

(a)

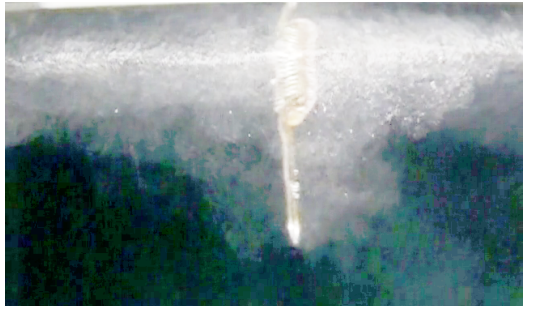

(d)

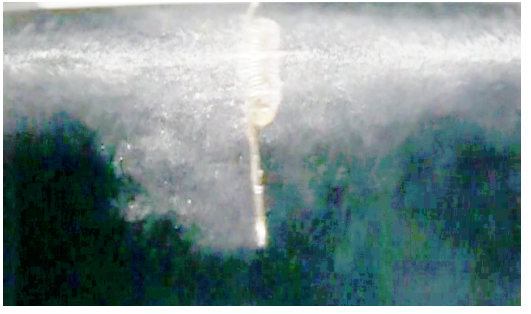

(g)

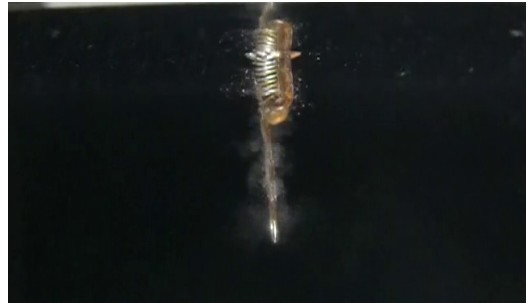

(b)

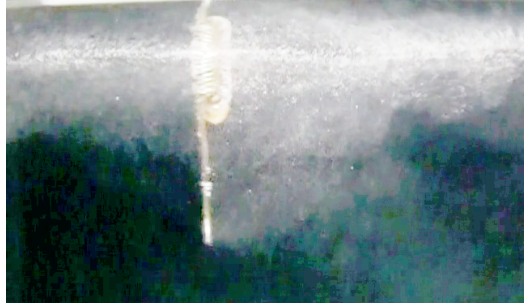

(e)

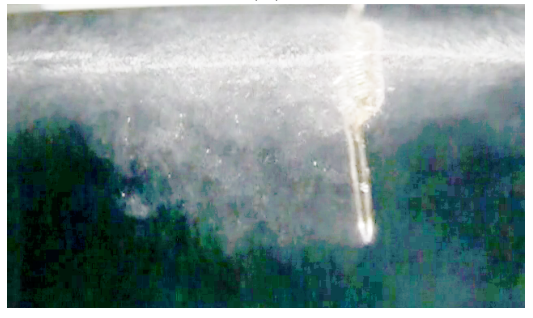

(h)

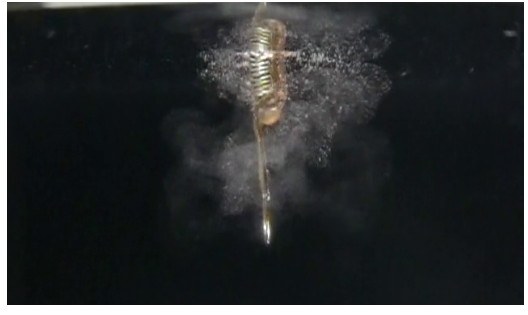

(c)

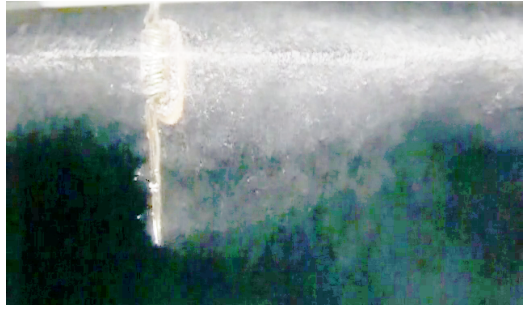

(f)

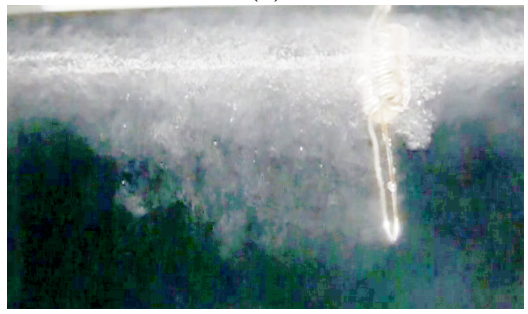

(i)

FIG. 2. (Color online) Photographs of the typical self-propelled swing motion due to the growing instability. (a) $t=0 \mathrm{~s}$; (b) $t=0.08 \mathrm{~s}$; (c) $t=0.46 \mathrm{~s}$; (d) $t=17.86 \mathrm{~s}$; (e) $t=17.95 \mathrm{~s}$; (f) $t=18.06$ $\mathrm{s} ;(\mathrm{g}) t=18.21 \mathrm{~s} ;$ (h) $t=18.31 \mathrm{~s} ;$ (i) $t=18.38 \mathrm{~s}$. Here, $V_{0}=7.7 \mathrm{~V}, I_{0}=9.5 \mathrm{~A}, P_{0}=73.2$ $\mathrm{W}, R_{0}=0.81 \Omega, q=4.77 \mathrm{MW} / \mathrm{m}^{2}, L=150 \mathrm{~mm}, L_{h}=9 \mathrm{~mm}, l_{h}=20 \mathrm{~mm}, \phi_{1}=0.26 \mathrm{~mm}$, $A=\pi \phi_{1} l_{h}=16.3 \mathrm{~mm}^{2}$, and $L_{w}=12 \mathrm{~mm}$.

cloud becomes large until $t=0.46 \mathrm{~s}$. Since the bubble cloud is rising upward, the cloud density difference in the upward direction indicates instability of the bubble generation in time, whereas the density difference between the right and left sides indicates instability of the bubble generation in position. Thus, from Fig. 2(c), we understand that this instability could be a trigger to start the swing motion. Figures 2(d) to 2(i) show the contrast-enhanced photographs during a large swing motion to clarify the relation between the bubble cloud and the motion of the heater. From these figures, we find that the swing motion of the heater is associated with the presence of an asymmetric bubble cloud, larger at the rear of the heater. Specifically, Figs. 2(d), 2(e), and 2(f) show the motion in the left direction 
$\left(\dot{X}_{p}<0\right)$ at $t=17.86,17.95$, and $18.06 \mathrm{~s}$, respectively, whereas Figs. 2(g), 2(h), and 2(i) show the motion in the right direction $\left(\dot{X}_{p}>0\right)$ at $t=18.21,18.31$, and 18.38 s, respectively. From Figs. 2(d) to 2(f), we clearly recognize that the bubble cloud on the right side of the heater is much stronger than that on the left side at $\dot{X}_{p}<0$, whereas the bubble cloud on the left side of the heater is much stronger than that on the right side at $\dot{X}_{p}>0$ as shown in Figs. 2(g) to 2(i). In summary, the motion of the heater is accompanied by a large bubble cloud in the direction opposing that of the motion of the pendulum.

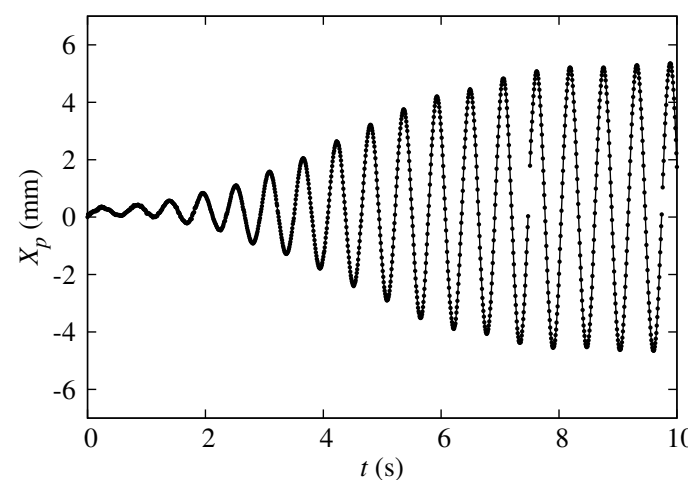

(a)

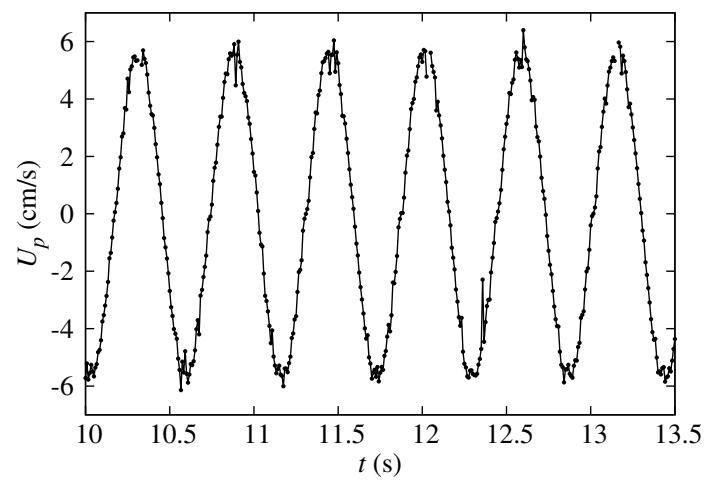

(c)

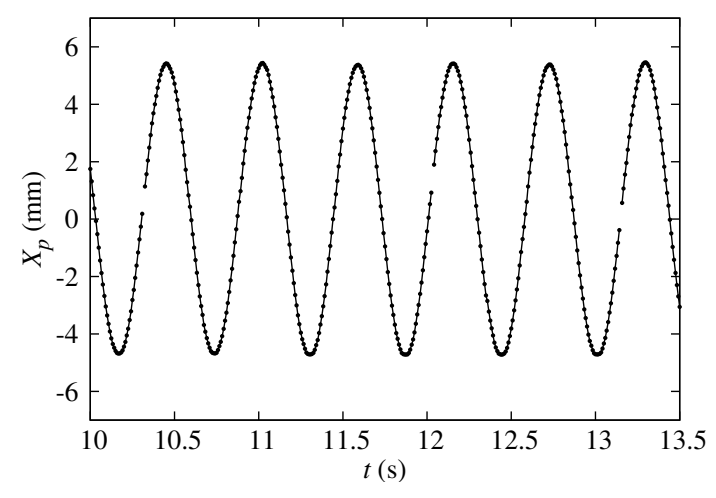

(b)

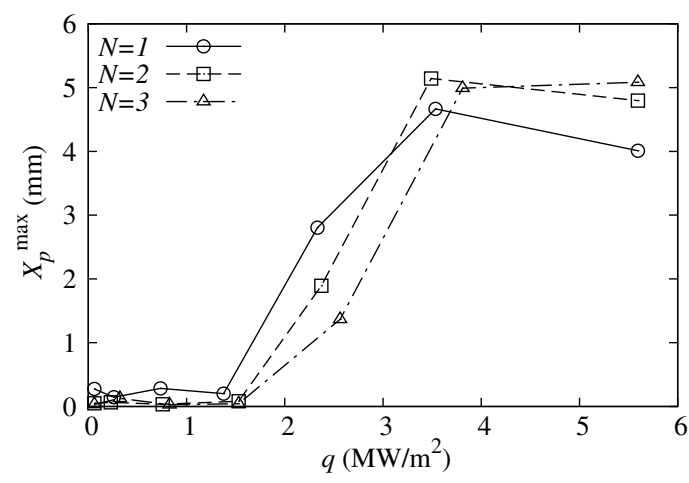

(d)

FIG. 3. Resonance characteristics of the self-propelled swing motion due to the growing instability. (a) Dependence of $X_{p}$ on $t$; (b) Dependence of $X_{p}$ on $t$; (c) Dependence of $U_{p}$ on $t$; (d) Dependence of $X_{p}^{\max }$ on $q$. Here, $L=100 \mathrm{~mm}, L_{h}=6 \mathrm{~mm}, l_{h}=16 \mathrm{~mm}, \phi_{1}=0.26 \mathrm{~mm}, A=\pi \phi_{1} l_{h}=13.1$ $\mathrm{mm}^{2}$, and $L_{w}=9 \mathrm{~mm}$; in (a) to (c), $N=3, V_{0}=7 \mathrm{~V}, I_{0}=10.3 \mathrm{~A}, P_{0}=73.1 \mathrm{~W}, R_{0}=0.68 \Omega$, and $q=5.6 \mathrm{MW} / \mathrm{m}^{2}$.

Figures 3(a) and 3(b) depict the dependence of $X_{p}$ on $t$, which corresponds to the swing motion. As shown in Fig. 3(a), the amplitude of $X_{p}$ initially increases with time finally to reach a constant value $(\sim 5 \mathrm{~mm})$. Here, the response time, which is the time to reach 
the steady state, is approximately $8 \mathrm{~s}$. The surprising aspect of this phenomenon is that the vibration starts on its own and is automatically stabilized. Furthermore, as shown in Fig. 3(b), $X_{p}$ shows a regular cyclic motion of frequency $f_{s}$ and amplitude $X_{p}^{\max }$ at $t \geq 10$ s, where $f_{s} \simeq 1.75 \mathrm{~Hz}$ and $X_{p}^{\max } \simeq 5.27 \mathrm{~mm}$ denote the frequency of this self-propelled motion and the maximum amplitude, respectively. It is surprising that a regular oscillation was produced from the stochastically irregular boiling phenomenon. In Fig. 3(b), if we assume that the swing motion at the steady state is a simple harmonic motion, it can be described as $X_{p}=X_{p}^{\max } \sin 2 \pi f_{s} t$. In this case, the velocity $U_{p}\left(\equiv \frac{d X_{p}}{d t}\right)$ is described as $U_{p}=2 \pi f_{s} X_{p}^{\max } \cos 2 \pi f_{s} t$. Figure $3(\mathrm{c})$ shows the dependence of $U_{p}$ on $t$. Here, the dependence corresponds to Fig. 3(b) and is obtained by numerical differentiation. Figure 3(c) indicates that the maximum velocity $\left(U_{p}^{\max }\right)$ of $U_{p}$ is approximately $6 \mathrm{~cm} / \mathrm{s}$; this agrees well with the expression $2 \pi f_{s} X_{p}^{\max }=5.8 \mathrm{~cm} / \mathrm{s}$. Thus, the self-swing motion can be treated as a simple harmonic motion, as the first approximation. Figure. 3(d) depicts the dependence of $X_{p}^{\max }$ on a heat flux $q=P_{0} / A$ applied on the heater. From Fig. 3(d), we find that the self-swing motion can be observed at approximately $q \geq 2.3 \mathrm{MW} / \mathrm{m}^{2}$ and exhibits a peak of $X_{p}^{\max }$ at $q \simeq 3.5 \mathrm{MW} / \mathrm{m}^{2}$.

To clarify the mechanism of this self-propelled swing motion, we performed several experiments. Figure 4 shows the results of additional experiments. Firstly, as shown in Fig. 4(a), the experimental frequency $f_{s, \exp }$ of the self-swing motion and the experimental natural frequency $f_{0, \exp }$ of the pendulum approximately agree with the theoretical curve of $f_{0, t h 1} \simeq \frac{1}{2 \pi} \sqrt{\frac{3 g}{2 L}}$, which will be derived from the discussion of Eq. (1) in Sec. IV.B. Thus, the self-swing motion is considered as a type of resonance phenomenon of the pendulum. Note that $f_{s, \exp }$ and $f_{0, \exp }$ are determined from the same experiment. For example, those values at $L=100 \mathrm{~mm}$ are determined from the steady oscillation part with $V_{0}=7 \mathrm{~V}$ at $t<17 \mathrm{~s}$ and the damped oscillation part with $V_{0}=0 \mathrm{~V}$ at $t>18 \mathrm{~s}$ in Fig. 4(b). Thus, their experimental conditions are completely same except $V_{0}$. Secondly, as shown in Fig. 4(c), the amplitude does not change significantly even if the tip region is bent to either the left or the right. Thus, the asymmetricity of the shape is irrelevant to the mechanism. Thirdly, as shown in Fig. $4(\mathrm{~d})$, the water temperature $T_{1}$ near the heater increases rapidly at approximately $q<0.7 \mathrm{MW} / \mathrm{m}^{2}$ compared to the water temperature $T_{2}$ at the bottom of the chamber. Furthermore, $T_{1}$ remains constant at approximately $q \geq 0.7 \mathrm{MW} / \mathrm{m}^{2}$. Here, we measured $T_{1}$ by placing the heater in contact with a thermocouple. The water just around the heater 
reaches its boiling temperature (i.e., $100{ }^{\circ} \mathrm{C}$ ) at $q \simeq 0.7 \mathrm{MW} / \mathrm{m}^{2}$ and attains a superheated state if the bubbles are not generated. Note that the self-swing motion is usually accompanied by a strong beep sound, which occurs due to an intense shock wave generated by the collapse of bubbles. In fact, we observed nucleate and film boiling with a self-swing motion, as shown in Fig. 2. In addition, $U_{p}^{\max }$ and $\theta^{\max }$ become large at approximately $q \geq 2.3$ $\mathrm{MW} / \mathrm{m}^{2}$, as shown in Figs. 4(e) and 4(f). Therefore, the self-swing motion occurs normally in the nucleate- to film-boiling regime and occurs strongly in the transition boiling regime.

\section{MODELS TO EXPLAIN SELF-SWING PHENOMENON}

\section{A. Qualitative explanation on our experiments}

Figure 5 shows the schematic of phenomenological model for the self-propelled swing motion. We consider that a small instability (or asymmetricity) of heat transfer between the left and right surfaces of the tip region grows into a large instability once the tip starts to move owing to the small instability. This is because the bubbles in the front of the hot tip region flow more than those in the rear and thus the temperature of the front becomes lower than that of the rear. As a result, the bubble generation probability at the front becomes lower than that of the rear, as shown in Figs. 5(a) and 5(b). Consequently, a larger amount of bubbles at the rear side generate a larger bubble force than the bubbles at the front side. Thus, the availability rate of the force generated by the bubble increases. Therefore, once the pendulum starts to swing, the amplitude increases with time and reaches a steady state when the increase in the velocity does not induce an increase in the amplitude (i.e., when the asymmetricity of heat transfer between the front and rear becomes maximum). Relative to a famous boiling curve, as shown in Fig. 5(c), the front region cannot move to the film boiling regime by passing from $C$ to $E$, and it remains in the nucleate boiling regime because the bubbles at the front flow out from the surface. Note that in Fig. 5(c), $q$ is the heat flux of the tip, $T_{h}$ is the temperature of the tip (heater), and $T_{f}$ is the temperature of the fluid far from the tip. 


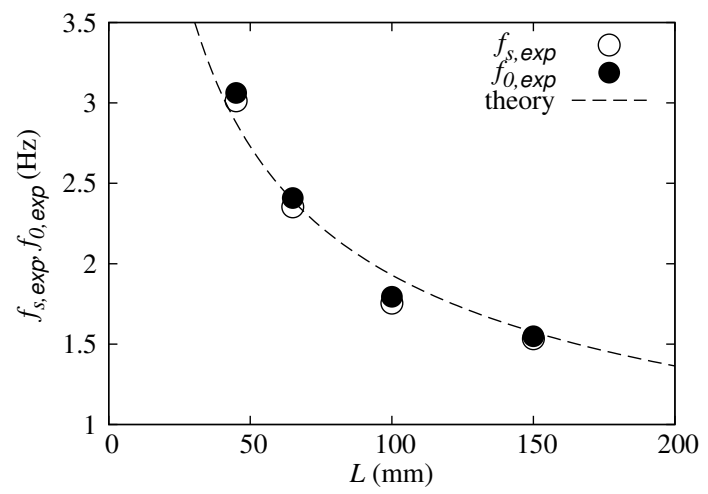

(a)

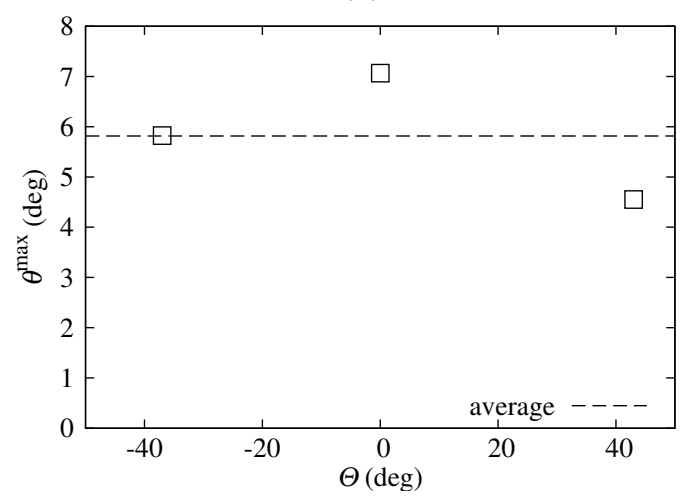

(c)

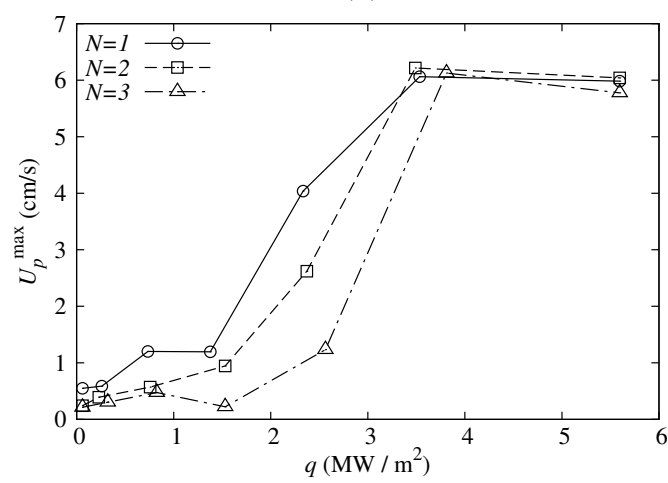

(e)

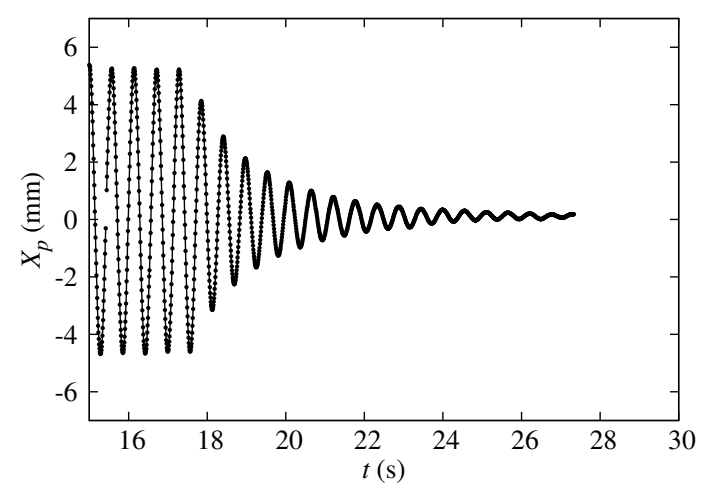

(b)

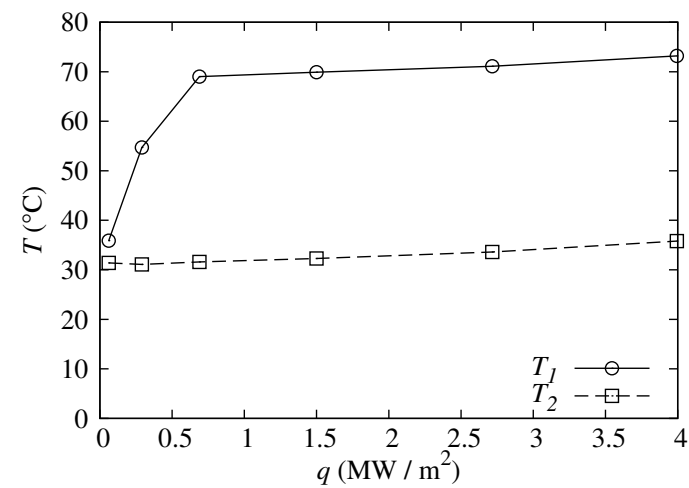

(d)

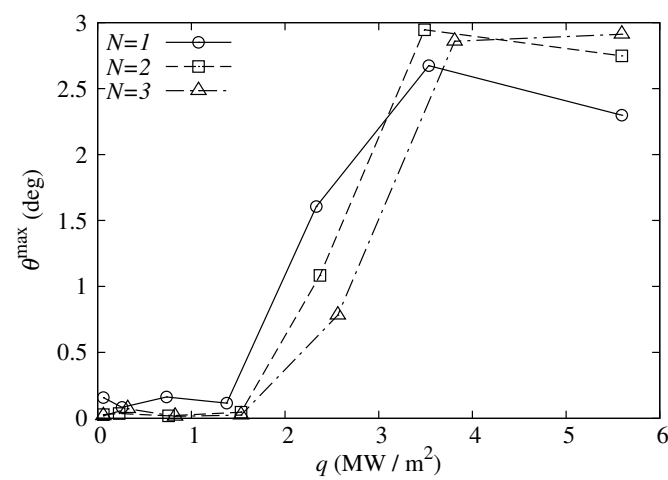

(f)

FIG. 4. Results for the additional experiments to clarify the mechanism of the self-propelled swing motion. (a) Dependences of $f_{0}$ and $f_{s}$ on $L$; (b) Experimental result for damped vibration; (c) Dependence of $\theta_{\max }$ on $\Theta\left(L=70 \mathrm{~mm}\right.$ and $\left.q=5.4 \mathrm{MW} / \mathrm{m}^{2}\right)$; (d) Dependencies of $T_{1}$ and $T_{2}$ on $q$ for the fixed pendulum $(\Theta=0 \mathrm{rad}$ and $L=90 \mathrm{~mm})$; (e) Dependence of $U_{p}^{\max }$ on $q(\Theta=0$ $\operatorname{rad}$ and $L=100 \mathrm{~mm})$; (f) Dependence of $\theta^{\max }$ on $q(\Theta=0 \operatorname{rad}$ and $L=100 \mathrm{~mm})$. 


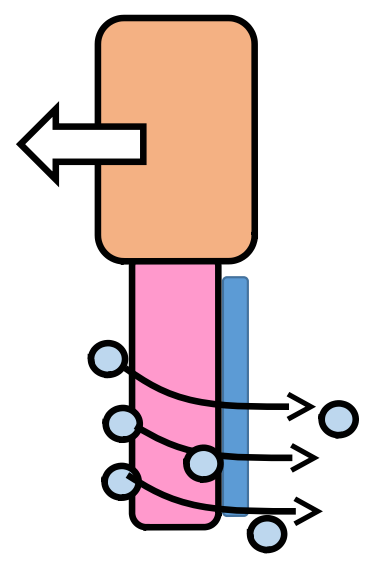

(a)

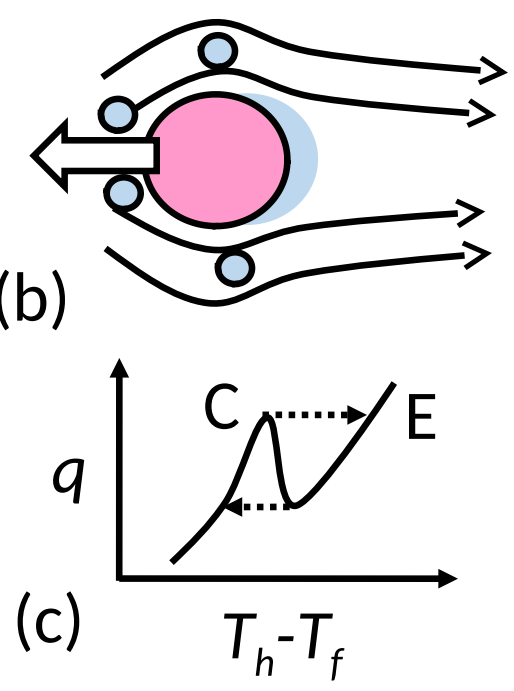

FIG. 5. (Color online) Schematic of our phenomenological model owing to the growing instability of asymmetrical heat transfer. (a) Side view of the tip region. (b) Top view of the tip region. (c) Boiling curve.

\section{B. Self-swing model with the superheat $\Delta T$ as a variable (Model A)}

From the above arguments, the self-swing phenomenon can be described by

$$
I \ddot{\theta} \simeq-\frac{m g L}{2} \theta+L f_{b}-\left(L-\frac{L_{w}}{2}\right) f_{v}
$$

where $m(1.2 \mathrm{~g}$ for the pendulum of $L=100 \mathrm{~mm})$ is the mass of the pendulum, $I=$ $\int_{0}^{L} r^{2} \frac{m}{L} d r=\frac{m L^{2}}{3}$ is the moment of inertia, $\theta \simeq \frac{X_{p}}{L}$ is a swing angle in Fig. 1 , and $f_{b}$ and $f_{v}$ are forces due to bubbles and viscosity, respectively. Note that from Eq. (1), the theoretical natural frequency is derived as $f_{0, t h 1}=\frac{1}{2 \pi} \sqrt{\frac{m g L}{2 I}} \simeq \frac{1}{2 \pi} \sqrt{\frac{3 g}{2 L}}$ since $I=\frac{m L^{2}}{3}$. When the Reynolds number $R e=\frac{\rho \dot{X_{p}} \phi_{1}}{\mu}$ of the system is less than $\sim 60$, the drag coefficient $c_{D}$ of the system is described by Stokes' formula $c_{D} \simeq \frac{24}{R e}[16,17]$, where $\rho$ and $\mu$ are the density and viscosity of water, respectively. Thus, $f_{v}$ is described by

$$
f_{v} \simeq c_{D} \rho \dot{X}_{p}^{2} A_{v}=12 \mu \frac{A_{v}}{\phi_{1}} \dot{X}_{p}
$$

where $A_{v}=\phi_{1} l_{h}+2\left(\phi_{2}+2 \phi_{1}\right)\left(L_{w}-L_{h}\right)$ is the cross-sectional area of the part immersed in water. Note that the nichrome wire was wrapped in a coil around the $\mathrm{Cu}$ wire as shown in Fig. 2(a). Thus, the diameter of that part above the heater is $\phi_{2}+2 \phi_{1}$. Further, $R e \simeq 1.6$ at 
most since $\dot{X}_{p}^{\max } \simeq 6 \mathrm{~mm} / \mathrm{s}$. Furthermore, as the first step, we phenomenologically assume that

$$
f_{b} \simeq \frac{A \Delta P}{2} R_{a} R_{b} \simeq \frac{A \Delta P}{2}\left(1-e^{-\frac{\dot{X_{p}}}{u_{c}}}\right)\left(1-e^{-\frac{\Delta T}{\Delta T_{c}}}\right)^{n}
$$

where $\Delta T$ is an average surface temperature deference (superheat) of the heater (which is measured from $T_{0}=373.15 \mathrm{~K}=100^{\circ} \mathrm{C}$ ),

$$
\Delta P(\Delta T)=P_{s}\left(T_{0}+\Delta T\right)-P_{s}\left(T_{0}\right)
$$

is an intrinsic pressure difference,

$$
P_{s}(T) \simeq p_{a} e^{-\frac{r_{0}}{R T}}
$$

is a saturated vapor pressure determined by the Clausius-Clapeyron equation (with the constant $p_{a}=0.1013 \mathrm{MPa}$, the evaporation latent heat $r_{0}=2256.9 \mathrm{~kJ} / \mathrm{kg}$, and the gas constant $R=0.4616 \mathrm{~kJ} / \mathrm{kg} \mathrm{K}$ of water),

$$
R_{a}\left(\dot{X}_{p}\right)=1-e^{-\frac{\dot{X_{p}}}{u_{c}}}
$$

is the availability rate of the force difference due to the broken symmetry of the right and left temperatures resulting from the non-zero value of $\dot{X}_{p}, u_{c}$ is a critical velocity causing a significant broken symmetry, and

$$
R_{b}=\left(1-e^{-\frac{\Delta T}{\Delta T_{c}}}\right)^{n}
$$

is an effective surface bubble coverage rate, which describes the surface bubble states from the nucleate- to film-boiling regime. Here, $u_{c}=5 \mathrm{~mm} / \mathrm{s}$ is selected to explain our experimental data, the characteristic temperature $\Delta T_{c}=60 \mathrm{~K}$ is selected approximately to be the Leidenfrost temperature, and the characteristic exponent $n=8$ is selected so that $\Delta P(170) \sim 4.5 \mathrm{MPa}$, which is known as the pressure of the film boiling. If $\frac{\dot{X}_{p}}{u_{c}} \ll 1$, we obtain $R_{a} \sim \frac{\dot{X}_{p}}{u_{c}}$. Thus, by using Eqs. (2) and (3), we can approximate Eq. (1) as

$$
\frac{m L}{3} \ddot{X}_{p} \simeq-\frac{m g}{2} X_{p}+A_{0} \dot{X}_{p}-B_{0} \dot{X}_{p}\left(a t \frac{\dot{X}_{p}}{u_{c}} \ll 1\right),
$$

where $A_{0} \simeq \frac{A L \Delta P}{2 u_{c}} R_{b}(>0)$ is the amplified factor for the driving force due to the asymmetrical heat transfer, and $B_{0} \simeq 12 \mu \frac{A_{v}}{\phi_{1}}\left(L-\frac{L_{w}}{2}\right)(>0)$ is the flow resistance factor due to the fluid viscosity of water. Therefore, if $A_{0} \geq B_{0}$ i.e.,

$$
\frac{A L \Delta P}{2 u_{c}} R_{b} \geq 12 \mu \frac{A_{v}}{\phi_{1}}\left(L-\frac{L_{w}}{2}\right),
$$


the amplified oscillation in Fig. 2(a) starts. Further, since the moment of force $N_{b}=L f_{b}$ due to bubbles in Eq. (1) becomes constant at $\frac{\dot{X}_{p}}{u_{c}} \gg 1$ (i.e., $N_{b} \simeq \frac{A L \Delta P}{2} R_{b}$ ), the steady oscillation in Fig. 2(b) appears. Furthermore, from Eqs.(7) and (9), the minimum of $u_{c}$ is described as

$$
u_{c}^{*}(\Delta T)=\frac{A L \Delta P}{24 \mu \frac{A_{v}}{\phi_{1}}\left(L-\frac{L_{w}}{2}\right)}\left(1-e^{-\frac{\Delta T}{\Delta T_{c}}}\right)^{n} .
$$

Since Eq. (9) is satisfied at $\Delta T(q) \geq \Delta T^{t h}\left(q^{t h}\right)$ for given $u_{c}, u_{c}^{*}\left(\Delta T^{t h}\right)$ is considered to be a reasonable candidate of $u_{c}$, where $\Delta T^{t h}$ and $q^{t h}$ are the threshold values of $\Delta T$ and $q$, respectively. Note that you can find more detail explanations on $R_{a}$ and $R_{b}$ in Appendix A.

\section{Model for $\Delta T(q)$ (Model B)}

From the experiments in the nucleate boiling regime, it is known that the heat-transfer coefficient $\alpha$ is proportional to the $2 / 3$ power of $q$ in the low heat flux region [15]. Thus, we assume that

$$
q=\alpha_{A}\left({\frac{q}{q_{A}}}^{\frac{2}{3}}\right) \Delta T
$$

where $\alpha_{A}$ denotes $\alpha$ at $q=q_{A}$ and $\Delta T=\Delta T_{A}$ (i.e., $\alpha_{A}=\frac{q_{A}}{\Delta T_{A}}$ ). By transforming Eq. (11), we obtain

$$
\Delta T=\frac{q}{\alpha_{A}\left(\frac{q}{q_{A}} \frac{2}{3}\right)}=\Delta T_{A}\left(\frac{q}{q_{A}}\right)^{\frac{1}{3}} .
$$

Therefore, by using Eq. (12) with Eq. (1), we can predict a motion at given $q$.

\section{The effects of parameters in Model A on fundamental values}

Figure 6 shows the effects of parameters in Model A. Specifically, Fig. 6(a) shows the dependence of $R_{a}$ on $U_{p}$ at $u_{c}=5,10$, and $15 \mathrm{~mm} / \mathrm{s}$. As shown in Fig. 6(a), although $R_{a}$ increases linearly in the initial stage $\left(U_{p} \ll u_{c}\right)$, the increasing rate slows down at $U_{p}>u_{c}$. This means that as $U_{p}$ increases, the temperature difference $\delta T$ between the forward and rear surfaces of the heater increases rapidly at $U_{p} \ll u_{c}$ but the increasing rate of $\delta T$ slows down at $U_{p}>u_{c}$ since $\delta T$ approaches $\Delta T$, which is the maximum value of $\delta T$. Figure $6(\mathrm{~b})$ shows the dependence of $u_{c}^{*}$ on $\Delta T$. As will be explained later, we obtained $\Delta T^{t h} \simeq 15 \mathrm{~K}$ and $q^{t h} \simeq 1.5 \mathrm{MW} / \mathrm{m}^{2}$. Thus, we set $u_{c}=5 \mathrm{~mm} / \mathrm{s}$ in this manuscript since $u_{c}^{*}=4.5 \mathrm{~mm} / \mathrm{s}$ at $\Delta T=15 \mathrm{~K}$ in Fig. 6(b). Figure 6(c) shows the dependence of $R_{b}$ on $\Delta T$ at $\Delta T_{c}=40,50$, 


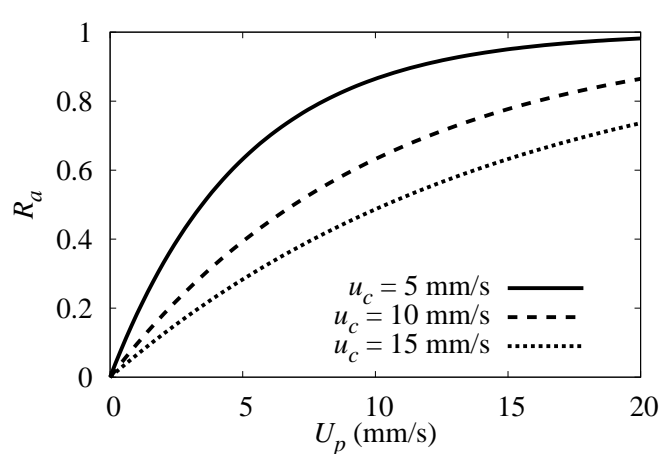

(a)

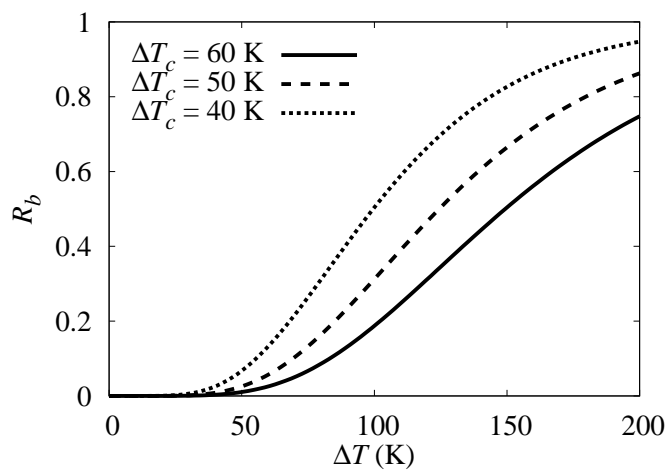

(c)

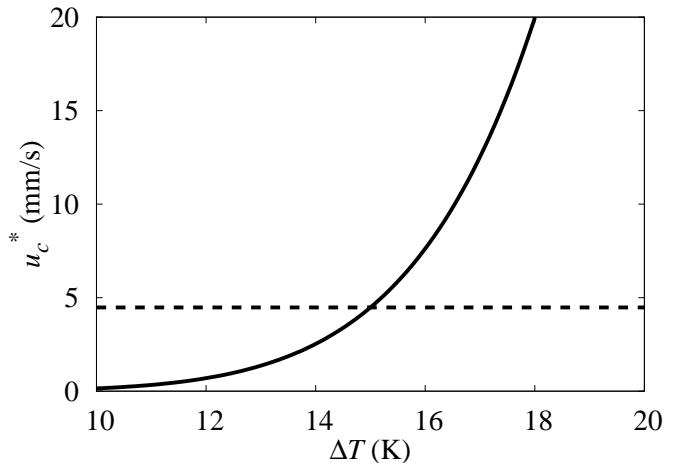

(b)

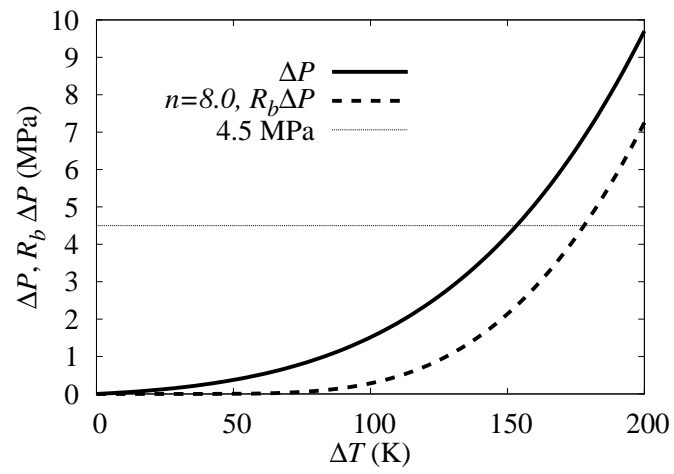

(d)

FIG. 6. The effects of parameters in Model A. (a) Dependence of $R_{a}$ on $U_{p}$; (b) Dependence of $u_{c}^{*}$ on $\Delta T$; (c) Dependence of $R_{b}$ on $\Delta T(n=8)$; (d) Dependences of $\Delta P$ and $R_{b} \Delta P$ on $\Delta T$. In (d), we use $\Delta T_{c}=60 \mathrm{~K}$ and $n=8$.

and $60 \mathrm{~K}$. As shown in Fig. 6(c), although $R_{b}$ increases rapidly at $\Delta T>\Delta T_{c}$. This means that the boiling phenomenon changes from the nucleate boiling state to the film boiling state at $\Delta T \sim \Delta T_{c}$. Although various values are reported for $\Delta T_{c}$, we use $\Delta T_{c}=60 \mathrm{~K}$ for our analysis, since we experimentally obtained $\Delta T_{c} \simeq 60 \mathrm{~K}$ in our Leidenfrost experiments [18]. Figure $6(\mathrm{~d})$ shows the dependences of $\Delta P$ and $R_{b} \Delta P$ on $\Delta T$ when $\Delta T_{c}=60 \mathrm{~K}$ and $n=8$. Even for a strong film boiling phenomenon, it is known that $\Delta P$ is an over estimated value for the initial pressure of the superheat layer of the bubble. For example, Asai et al. [19] reported that the initial pressure of the superheat layer is $\sim 4.5 \mathrm{MPa}$ at $\Delta T \sim 170 \mathrm{~K}$. Thus, we set $n=8$ to satisfy this condition approximately. As shown in Fig. 6(d), $R_{b} \Delta P$ significantly increases from the Leidenfrost temperature $(\Delta T \simeq 60 \mathrm{~K})$ and becomes $4.5 \mathrm{MPa}$ at $\Delta T \simeq 180 \mathrm{~K}$. Here, $R_{b} \Delta P$ just shows the possible maximum pressure. In other words, only when $R_{a} \neq 0$ (i.e., only when $\dot{X}_{p} \neq 0$ ), it can accelerate the pendulum. Therefore, 
although the origin of the propulsion is $R_{b} \Delta P$, what causes propulsion is $R_{a}\left(\dot{X}_{p}\right)$ and the trigger is the instability of the bubble generation [in Fig. 2(c)]. Thus, we assumed a small initial velocity $\left(\dot{X}_{p}=10 \mu \mathrm{m} / \mathrm{s}\right.$ at $\left.t=0 \mathrm{~s}\right)$ as an initial initial instability in the calculations through our manuscript.

\section{E. Theoretical results using Model A for self-swing motions}

Figure 7 shows the theoretical analysis using Model A for self-swing motions. Here, we calculate the second order differential equation of Eq. (1) under the constant $\Delta T$ and $u_{c}$. In detail, we transform Eq. (1) as follows:

$$
\begin{array}{r}
\dot{X}_{p}=U_{p}, \\
\dot{U}_{p}=-C X_{p}+f\left(X_{p}, U_{p}\right), \\
C=\frac{m g L}{2 I}, \\
f\left(X_{p}, U_{p}\right)=\frac{L}{I}\left[L f_{b}-\left(L-\frac{L_{w}}{2}\right) f_{v}\right]
\end{array}
$$

and solved them by Euler method. That is, we calculate the difference equations

$$
\begin{array}{r}
U_{p}^{n+1}=U_{p}^{n}+\Delta t\left[-C X_{p}^{n}+f\left(X_{p}^{n}, U_{p}^{n}\right)\right] \\
X_{p}^{n+1}=X_{p}^{n}+\Delta t U_{p}^{n+1}
\end{array}
$$

with the initial conditions that $X_{p}^{0}=X_{p}(t)=0 \mathrm{~mm}$ and $U_{p}^{0}=U_{p}(0)=10 \mu \mathrm{m} / \mathrm{s}$, where $\Delta t$ is a time interval, the superscript $n$ indicates the time step, and $t=n \Delta t$. Note that $U_{p}(0)=10$ $\mu \mathrm{m} / \mathrm{s}$ is assumed to represent the initial fluctuation due to the boiling phenomenon under consideration.

As shown in Figs. 7(a) and 7(b), we find that the increasing of $\Delta T$ mainly results in the increasing of $X_{p}^{\max }$, whereas the increasing of $u_{c}$ results in the increasing of the response time $\tau_{1}$. Here, we define $\tau_{1}$ as $\tau_{1} \equiv t_{2}-t_{1}$, where $t_{1}$ and $t_{2}$ satisfy the condition that $X_{p}\left(t_{1}\right)=0.05 X_{p}^{\max }$ and $X_{p}\left(t_{2}\right)=0.95 X_{p}^{\max }$. In detail, $X_{p}^{\max }$ and $\tau_{1}$ depends on $\Delta T$ and $u_{c}$, as shown in Figs. $7(\mathrm{c})$ to $7(\mathrm{f})$. Since $u_{c}$ is considered to be approximately $5 \mathrm{~mm} / \mathrm{s}$ as mentioned before, we can understand that Model A is justified if we can predict $X_{p}$ and $\tau_{1}$ well at the same time for our experiments by setting suitable $\Delta T$. 


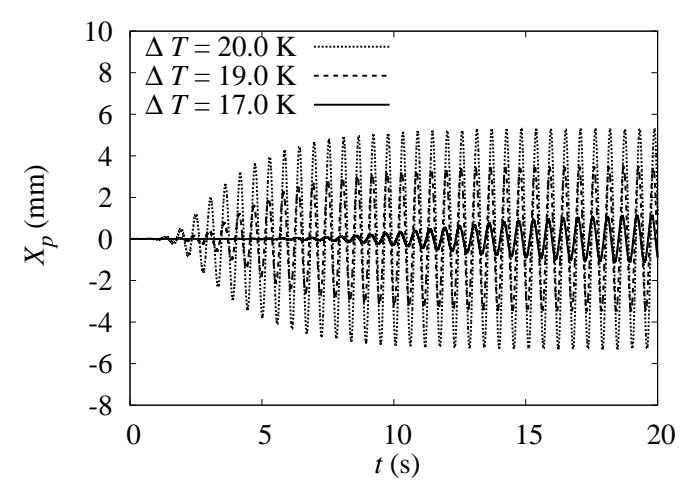

(a)

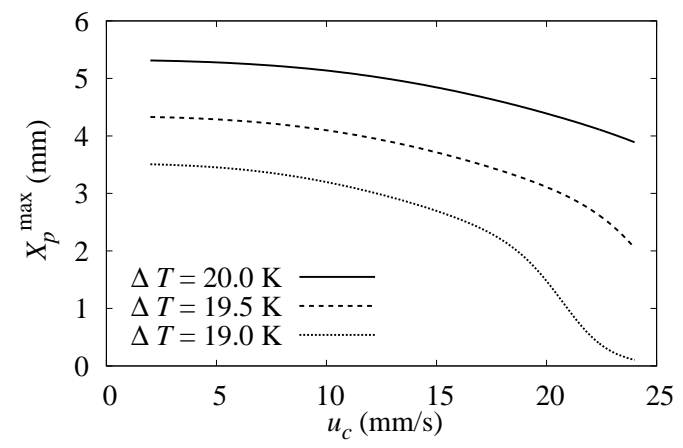

(c)

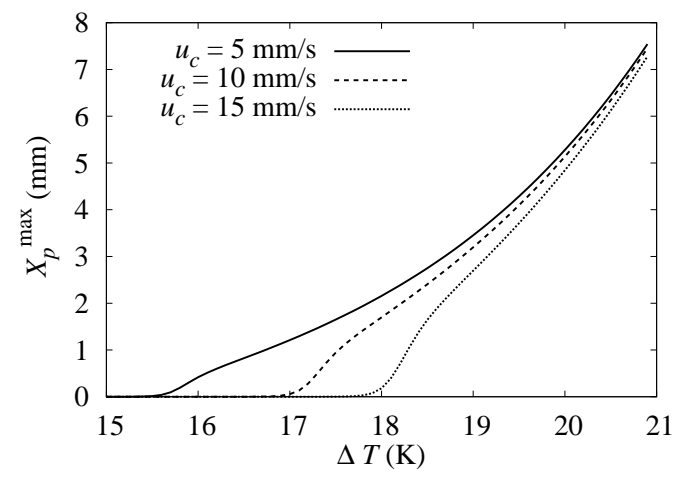

(e)

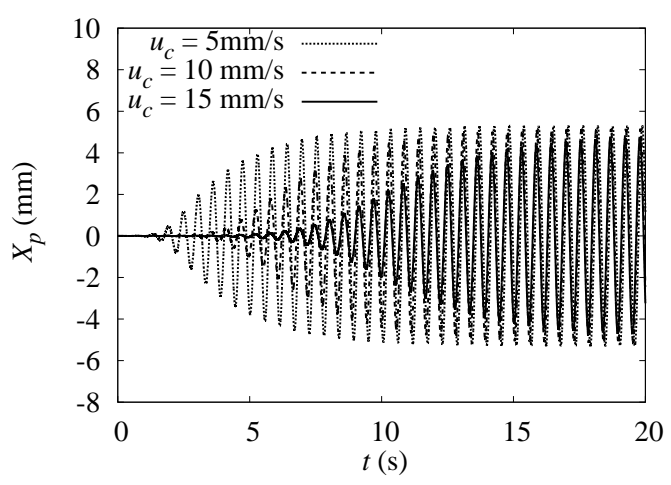

(b)

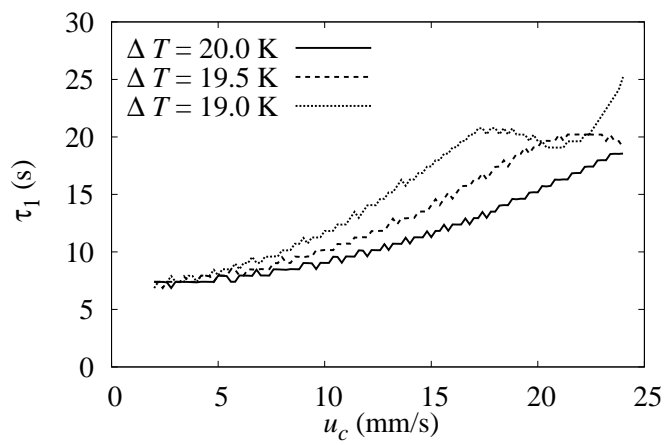

(d)

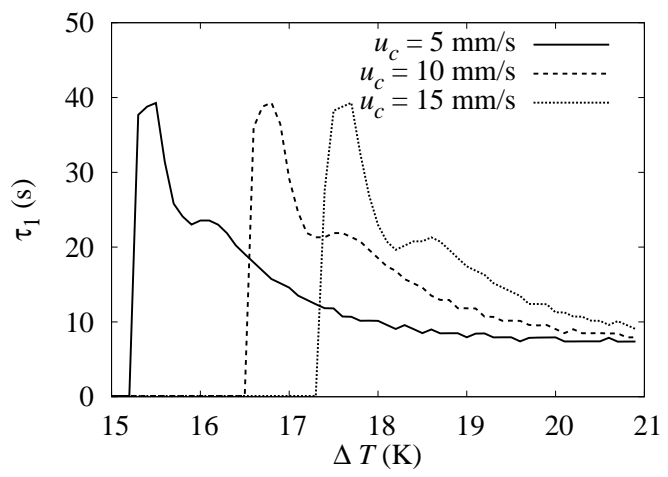

(f)

FIG. 7. Theoretical results using Model A for self-swing motions. (a) Dependence of $X_{p}$ on $t$ at $u_{c}=5 \mathrm{~mm} / \mathrm{s}$; (b) Dependence of $X_{p}$ on $t$ at $\Delta T=20 \mathrm{~K}$; (c) Dependence of $X_{p}^{\max }$ on $u_{c}$; (d) Dependence of $\tau_{1}$ on $u_{c}$; (e) Dependence of $X_{p}^{\max }$ on $\Delta T$; (f) Dependence of $\tau_{1}$ on $\Delta T$. Here, $L=100 \mathrm{~mm}, L_{h}=6 \mathrm{~mm}, l_{h}=16 \mathrm{~mm}, \phi_{1}=0.26 \mathrm{~mm}, A=\pi \phi_{1} l_{h}=13.1 \mathrm{~mm}^{2}$, and $L_{w}=9 \mathrm{~mm}$; $\mu=1.0 \mathrm{mPa} \mathrm{s}, \Delta T_{c}=60 \mathrm{~K}$, and $n=8$. 


\section{ANALYSIS USING THE THEORETICAL AND EXPERIMENTAL RESULTS}

\section{A. Analysis for the dependence on $q$}

Figure 8 shows the comparison between the experimental results and the theoretical result under the condition that $L=100 \mathrm{~mm}, L_{h}=6 \mathrm{~mm}, l_{h}=16 \mathrm{~mm}, \phi_{1}=0.26 \mathrm{~mm}$, $A=\pi \phi_{1} l_{h}=13.1 \mathrm{~mm}^{2}$, and $L_{w}=9 \mathrm{~mm}$. Specifically, Fig. 8(a) shows the comparison for the damped oscillation when $q=5.6 \mathrm{MW} / \mathrm{m}^{2}$ was shut off. Here, we assume $I^{*}=1.17 I$ instead of $I$ in Eq. (1) so that the frequency of the numerical calculation well corresponds to that of the experiment. Note that there is no free parameter for $f_{0, t h 1}$ since it is determined by the partial part $I \ddot{\theta} \simeq-\frac{m g L}{2}$ of Eq. (1). Thus, $I^{*}=1.17 I$ must be adopted as a true value of $I$. Furthermore, we use $\mu=1.0 \mathrm{mPa}$ s for our calculations as the typical value of water at room temperature. This is probably justified as the first step since the water was mixed in the whole region by the swing motion of the pendulum. In fact, we need not adjust $\mu$ so that the decay time of the numerical calculation well corresponds to the experiments. That is, as shown in Fig. 8(a), the experimental results of the damped oscillation agrees well with the theoretical results provided by Model A not only for the frequency but also for the decay time. Since $\mu$ is the only free parameter for $I^{*} \ddot{\theta} \simeq-\frac{m g L}{2}-\left(L-\frac{L_{w}}{2}\right) f_{v}$, our model is reliable at least for the viscosity term (i.e., the $f_{v}$ term). That is, Eqs. (1) and (2) are justified except the $f_{b}$ term described in Eq. (3).

Figures $8(\mathrm{~b}), 8(\mathrm{c})$, and $8(\mathrm{~d})$ show the comparison for the resonance vibrations at $q=5.6$, 3.8, and $2.5 \mathrm{MW} / \mathrm{m}^{2}$, respectively under the same condition except $q$. Here, for Model A, we assume $\Delta T=20.0,20.0$, and 17.0 for $q=5.60,3.81$, and $2.56 \mathrm{MW} / \mathrm{m}^{2}$, respectively, so that the amplitudes of the numerical results well correspond to those of experiments. Note that $\Delta T$ is the only parameter for Eq. (1), if we admit that $I^{*}=1.17 I$ and $\mu=1.0$ $\mathrm{mPa} \mathrm{s}$ with the conditions that $\Delta T_{c}=60 \mathrm{~K}, u_{c}=5 \mathrm{~mm} / \mathrm{s}$, and $n=8$, which conditions are determined from the arguments in Sec. IV.D. As shown in Figs. 8(b), 8(c), and 8(d), the experimental results of the resonance vibration agrees fairly well with the theoretical results provided by Model A not only for the amplitudes but also for the response times. Thus, the $f_{b}$ term is also justified; i.e., Model A described Eqs. (1) to (7) are justified. That is, our model predicts that the amplitude of the pendulum increases with time and finally reaches a constant value (which depends on the power). In other words, our model predicts 


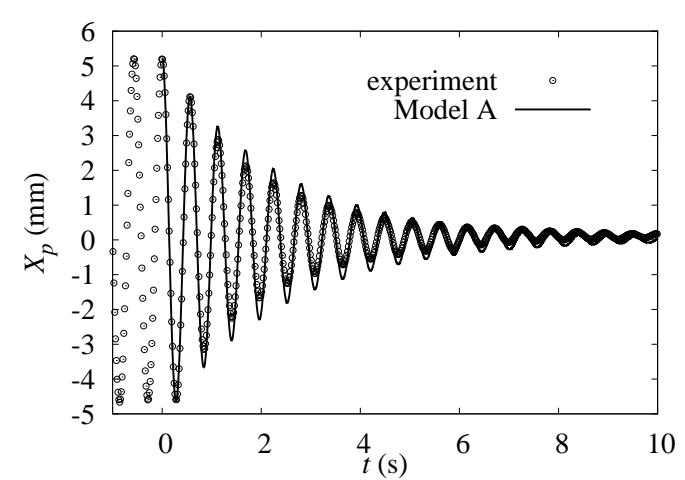

(a)

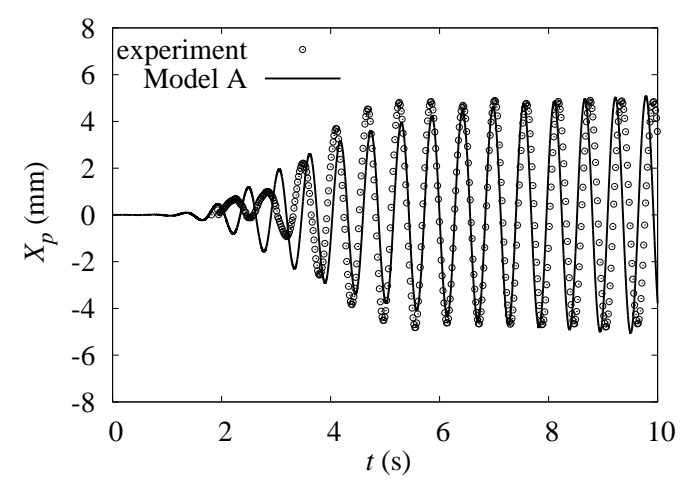

(c)

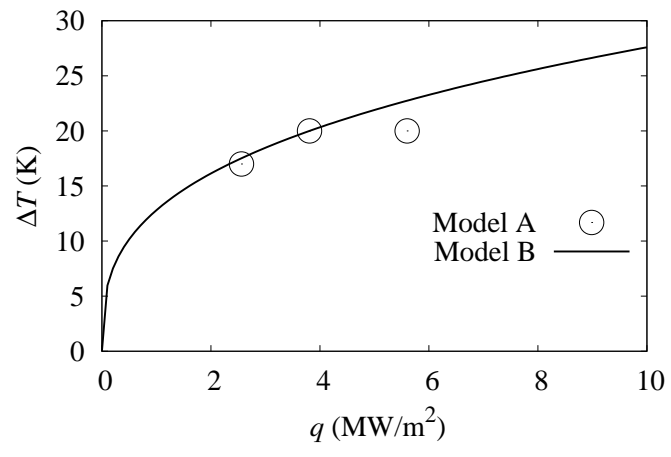

(e)

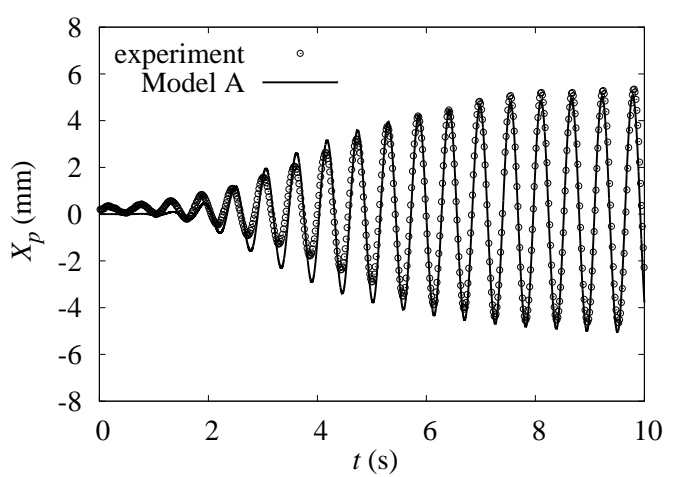

(b)

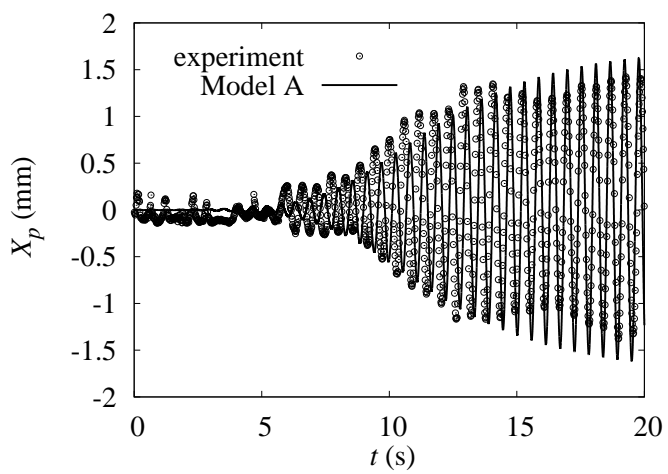

(d)

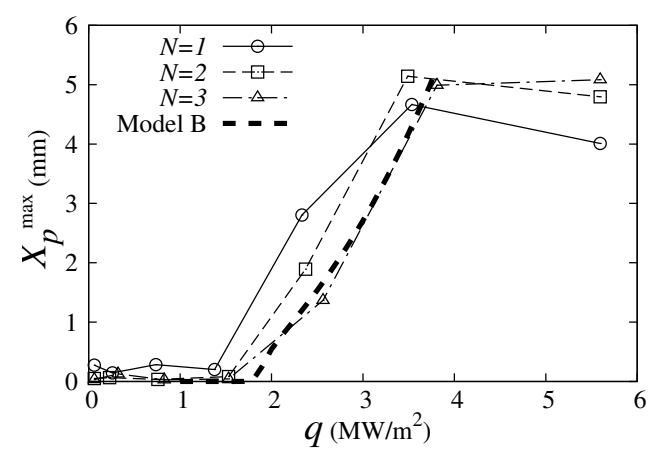

(f)

FIG. 8. Comparison between the experimental results and the theoretical results for the dependences on $q$. (a) Comparison for damped vibration when $q=5.60 \mathrm{MW} / \mathrm{m}^{2}$ was shut off; (b) Comparison for resonance vibration at $q=5.60 \mathrm{MW} / \mathrm{m}^{2}$; (c) Comparison for resonance vibration at $q=3.81 \mathrm{MW} / \mathrm{m}^{2}$; (d) Comparison for resonance vibration at $q=2.56 \mathrm{MW} / \mathrm{m}^{2}$; (e) Dependence of $\Delta T$ on $q$; (f) Comparison for the dependence of $X_{p}^{\max }$ on $q$. Here, the geometrical conditions are $L=100 \mathrm{~mm}, L_{h}=6 \mathrm{~mm}, l_{h}=16 \mathrm{~mm}, \phi_{1}=0.26 \mathrm{~mm}, A=\pi \phi_{1} l_{h}=13.1 \mathrm{~mm}^{2}$, and $L_{w}=9 \mathrm{~mm} ;$ in (a) to (e), $N=3$; in (b), (c), and (d), we assume that $\Delta T=20.0,20.0$, and $17.0 \mathrm{~K}$, respectively; the conditions for the theoretical results are $\mu=1.0 \mathrm{mPa} \mathrm{s}, \Delta T_{c}=60 \mathrm{~K}, u_{c}=5 \mathrm{~mm} / \mathrm{s}$, and $n=8$. 
experimental results very well.

Figure 8(e) shows the comparison between Models $\mathrm{A}$ and $\mathrm{B}$ for the dependence of $\Delta T$ on $q$. Here, we assume $\Delta T_{A}=20.0 \mathrm{~K}, q_{A}=3.81 \mathrm{MW} / \mathrm{m}^{2}$, and $\alpha_{A}=0.191 \mathrm{MW} /\left(\mathrm{m}^{2} \mathrm{~K}\right)$ for Model B. As shown in Fig. 8(e), $\Delta T$ values obtained by Model A is consistent with those obtained by Model B at $q \leq 3.81 \mathrm{MW} / \mathrm{m}^{2}$. That is, $\Delta T$ at $q=2.56 \mathrm{MW} / \mathrm{m}^{2}$ is predicted well by Eq. (12). Note that there is no free parameter for this comparison once we admit that $\Delta T_{A}=20.0 \mathrm{~K}$ and $q_{A}=3.81 \mathrm{MW} / \mathrm{m}^{2}$, which values are determined from Fig. $8(\mathrm{c})$. Furthermore, Fig. 6(f) shows the comparison between the experimental results of $X_{p}^{\max }$ and the theoretical results obtained by Model B coupled with Model A for the dependence of $\Delta X_{p}^{\max }$ on $q$. As shown in Fig. 8(f), the experimental results of the damped oscillation agrees well with the theoretical results provided by Model B coupled with Model A at $q \leq 3.81$ $\mathrm{MW} / \mathrm{m}^{2}$. Note that there is also no free parameter for this comparison once we admit that $\Delta T_{A}=20.0 \mathrm{~K}$ and $q_{A}=3.81 \mathrm{MW} / \mathrm{m}^{2}$ and thus our model is reliable. In particular, the fact that the experimental threshold value $q_{t h}^{e x p}$ agrees well with the theoretical threshold value $q_{t h}^{\text {model } B}$ justifies the assumption that $u_{c}=5 \mathrm{~mm} / \mathrm{s}$. From those analyses, Models A and B are justified, although we need to improve Model B at $q>3.81 \mathrm{MW} / \mathrm{m}^{2}$.

\section{B. Analysis for the dependence on $L$}

Figure 9 shows the comparison between the experimental and theoretical results for the dependences on $L$ at $q \simeq 5.6 \mathrm{MW} / \mathrm{m}^{2}$ (in detail, $q=5.40$ to $5.63 \mathrm{MW} / \mathrm{m}^{2}$ ). Here, the geometrical conditions are $L_{h}=6.0$ to $7.2 \mathrm{~mm}, l_{h}=15.0$ to $17.0 \mathrm{~mm}, \phi_{1}=0.26 \mathrm{~mm}$, and $L_{w}=9.0$ to $10.8 \mathrm{~mm}$; the conditions for the theoretical results are $\mu=0.7974 \mathrm{mPa} \mathrm{s}$, $\Delta T=20.0$ or $21.8 \mathrm{~K}, \Delta T_{c}=60 \mathrm{~K}, u_{c}=5 \mathrm{~mm} / \mathrm{s}$, and $n=8$. Specifically, Figs. 9(a), 9(b), and $9(\mathrm{c})$ show the comparison for the dependences of $X_{p}^{\max }, U_{p}^{\max }$, and $\theta^{\max }$, respectively. As shown in those figures, Model A captures the behavior of $X_{p}^{\max }, U_{p}^{\max }$, and $\theta^{\max }$ to some extent, although there exist variations in experimental data corresponding to the difference of $\Delta T=20.0$ to $21.8 \mathrm{~K}$. Furthermore, Fig. $9(\mathrm{~d})$ shows the comparison for the dependences of $X_{p}$ and $U_{p}$ on $t$ at $L=100 \mathrm{~mm}$ in the initial stage $(t \leq 4)$. As shown in Fig. 9(d), Model A captures the initial behaviors of $X_{p}$ and $U_{p}$ on $t$ to some extent, although we may need to improve our model more in the future. 


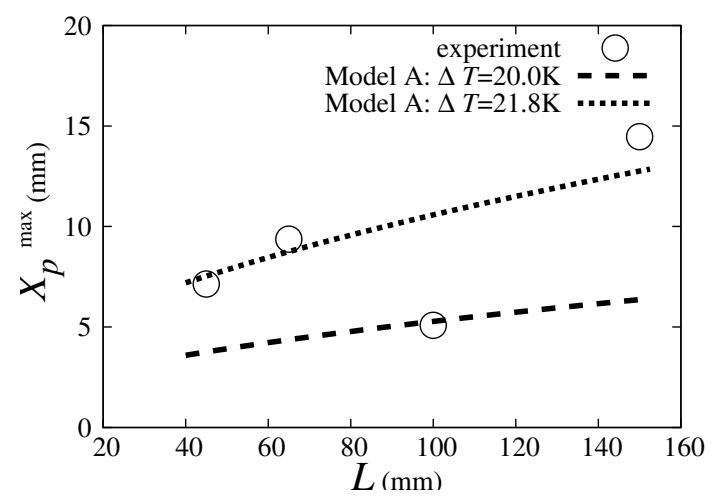

(a)

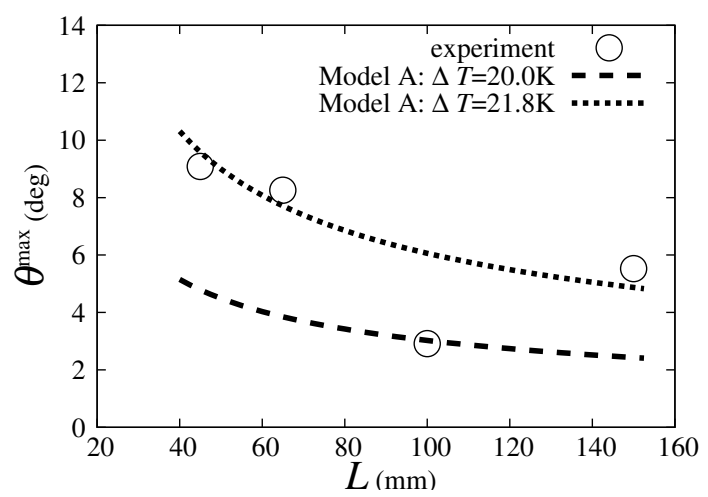

(c)

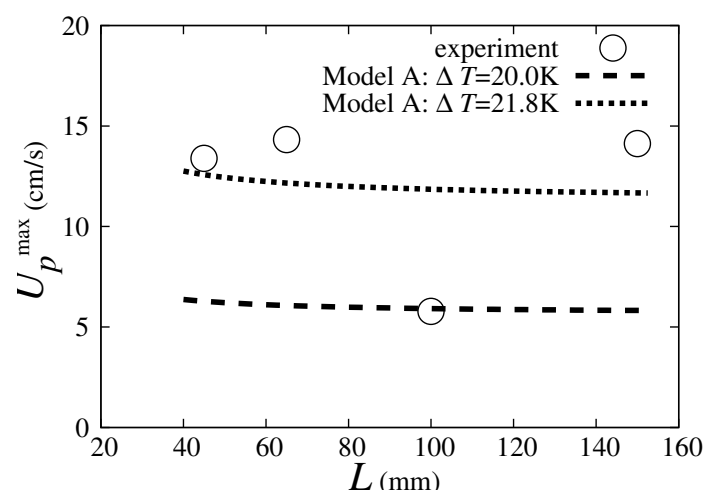

(b)

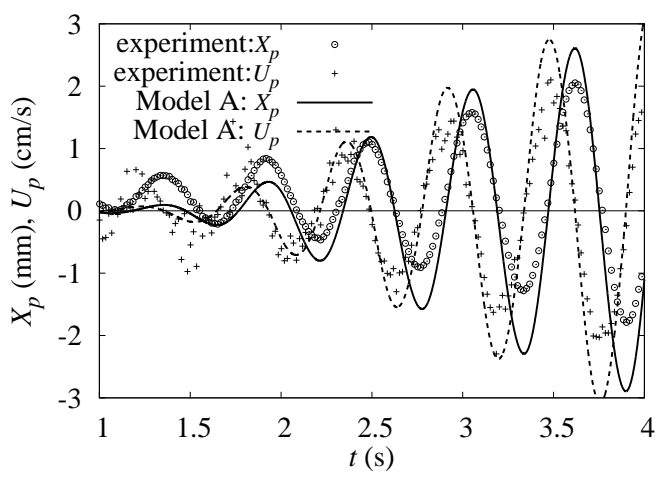

(d)

FIG. 9. Comparison between the experimental and theoretical results for the dependences on $L$ at $q \simeq 5.6 \mathrm{MW} / \mathrm{m}^{2}$ (in detail, $q=5.40$ to $5.63 \mathrm{MW} / \mathrm{m}^{2}$ ). (a) Comparison for the dependence of $X_{p}^{\max }$ on $L$; (b) Comparison for the dependence of $U_{p}^{\max }$ on $L$; (c) Comparison for the dependence of $\theta^{\max }$ on $L$; (d) Comparison for the dependences of $X_{p}$ and $U_{p}$ on $t$ at $L=100 \mathrm{~mm}$. Here, the geometrical conditions are $L_{h}=6.0$ to $7.2 \mathrm{~mm}, l_{h}=15.0$ to $17.0 \mathrm{~mm}, \phi_{1}=0.26 \mathrm{~mm}$, and $L_{w}=9.0$ to $10.8 \mathrm{~mm}$; the conditions for the theoretical results are $\mu=1.0 \mathrm{mPa} \mathrm{s}, \Delta T=20.0$ or $21.8 \mathrm{~K}$, $\Delta T_{c}=60 \mathrm{~K}, u_{c}=5 \mathrm{~mm} / \mathrm{s}$, and $n=8$.

\section{DISCUSSION}

\section{A. Meaning of our study}

In this study, we first reported that a pendulum having a hot tip exhibits a strong selfswing motion at a heat flux of approximately $3.5 \mathrm{MW} / \mathrm{m}^{2}$. Surprisingly, in contrast to Linke et al.'s device and other similar devices, this new self-swing device does not require sudden contact with a temperature exceeding the Leidenfrost temperature or with a suddenly 
applied extremely large heat flux of $\sim 1 \mathrm{GW} / \mathrm{m}^{2}$. In other words, the self-swing device can start even at room temperature by gradually increasing the heat flux of the tip region. This characteristic is useful for developing energy conversion systems that utilize waste heat as their energy source. For example, we consider the heat sink having plural pendulums instead of fins. In this sink, the pendulums are immersed in water and have magnetism. By preparing coils near the pendulums, we can convert heat energy, which is transmitted from the base of the heat sink, into electrical energy. Here, we can use relatively low grade thermal energies such as waste heat energy exhausted from factories and power plants, heat energy of iron plate heated by the sun in the summer, etc. Although the temperature of such low energy sauces usually fluctuates during the day, pendulums can start to move when their temperature reaches boiling temperature and can convert thermal energy into electrical energy. Furthermore, our device can be used to biomedical microfluidic devices as a new class of self-propelled micro heat engine. For example, by replacing the pendulums with oblique elastic beams, we can make new artificial cilia (which is expected as a next-generation multi-functional microfluidic device [20]) and can realize a new outstanding micropump (or microswimmer) whose velocity may exceed $5 \mathrm{~cm} / \mathrm{s}$ (which is approximately 37 times larger than that of the state of the art [21]) in the future. Thus, our finding is useful not only for energy harvesting techniques but also for biomedical microfluidic applications.

\section{B. Reasons why our models are justified}

As explained above, there are several reasons that justify our models. That is, (i) Eq. (1) is justified under the condition $f_{a}=f_{b}=0$, since our observed swing motions are recognized as a resonance phenomenon from Fig. 4(a). (ii) Eq. (2) is justified from Fig. 8(a). Thus, there is no doubt about $f_{v}$. (iii) Eqs. (3) to (7) are justified from Figs. 8(b) to 8(d); i.e., a bubble force term $f_{b}$ is justified. In particular, since the shape of the resonance oscillation is complex, it is difficult to match $X_{p}^{\max }$ and $\tau_{1}$ with the experimental values at the same time, if Model A is not correct. Thus, Model A is reliable. (iv) The choice of $u_{c}=5 \mathrm{~mm} / \mathrm{s}$ is justified since $u_{c}^{*}\left(\Delta T^{t h}\right) \sim 5 \mathrm{~mm} / \mathrm{s}$ since $q^{t h} \sim 1.5 \mathrm{MW} / \mathrm{m}^{2}$ and $\Delta T^{t h} \sim 15 \mathrm{~K}$ from Figs. $8(\mathrm{e})$ and $8(\mathrm{f})$. In other words, our models are self-consistent in our analysis. (v) The predicted $\Delta T$ using Model A [i.e., $\Delta T=15$ to $20 \mathrm{~K}$ for $q=2.56$ to $5.60 \mathrm{MW} / \mathrm{m}^{2}$ in Fig. $8(\mathrm{e})$ ] are reasonable as the values in the transition region [22]. That is, our models are consistent 


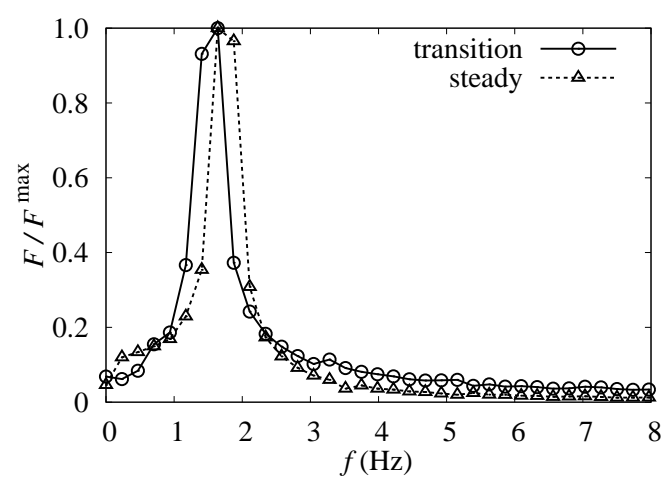

FIG. 10. Fourier analysis on the self-propelled swing motion. Here, open circles show the results for the oscillation during transition state $(0 \leq t \leq 8.5 \mathrm{~s})$ in Fig. 3(a), while open triangles show the results for the oscillation during steady state $(10.0 \leq t \leq 14.3 \mathrm{~s})$ in Fig. $3(\mathrm{~b}) ; n=512$, $\Delta t=0.008333 \mathrm{~s}, \Delta f=\frac{1}{n \Delta t}=0.234 \mathrm{~Hz} ; L=100 \mathrm{~mm}, L_{h}=6 \mathrm{~mm}, l_{h}=16 \mathrm{~mm}, \phi_{1}=0.26 \mathrm{~mm}$, $A=\pi \phi_{1} l_{h}=13.1 \mathrm{~mm}^{2}$, and $L_{w}=9 \mathrm{~mm} ; N=3, V_{0}=7 \mathrm{~V}, I_{0}=10.3 \mathrm{~A}, P_{0}=73.1 \mathrm{~W}, R_{0}=0.68$ $\Omega$, and $q=5.6 \mathrm{MW} / \mathrm{m}^{2}$.

with results from other literature. (vi) Model B is justified from Figs. 8(e) and 8(f). In particular, Model B used with Model A depicts the dependence of $X_{p}^{\max }$ on $q$ clearly at $q \leq 3.81 \mathrm{MW} / \mathrm{m}^{2}$. Note that as shown in Figs. 8(e) and $8(\mathrm{f}), \Delta T$ and $X_{p}^{\max }$ are almost constant at $q \geq 3.81 \mathrm{MW} / \mathrm{m}^{2}$; i.e., $\Delta T \sim 20 \mathrm{~K}$ and $X_{p}^{\max } \sim 5 \mathrm{~mm} / \mathrm{s}$ at $q \geq 3.81 \mathrm{MW} / \mathrm{m}^{2}$. Although it means that results of Model $\mathrm{B}$ do not agree with the experimental values at $q \geq 3.81 \mathrm{MW} / \mathrm{m}^{2}$, we consider that this is the evidence that the phenomenon occurs in the transition boiling region. In other words, we consider that our self-swing motion occurs near the $\mathrm{C}$ point in the boiling curve in Fig. $5(\mathrm{c})$ at $q \geq 3.81 \mathrm{MW} / \mathrm{m}^{2}$. From those arguments, we consider that our models described by Eq. (1) to (7), (10), and (12) are justified definitely.

\section{About the Fourier analysis on the oscillation}

Figure 10 shows the Fourier analysis on the self-propelled swing motion. That is, we use the fast Fourier transform (FFT) of the sample number $n=512$, while $F$ shows the result of FFT for $X_{p}(t)$. In Fig. 10, open circles show the results for the oscillation during the transition state $(0 \leq t \leq 8.5 \mathrm{~s})$ in Fig. $3(\mathrm{a})$, while open triangles show the results for the oscillation during the steady state $(10.0 \leq t \leq 14.3 \mathrm{~s})$ in Fig. 3(b). As shown in Fig. 10, 


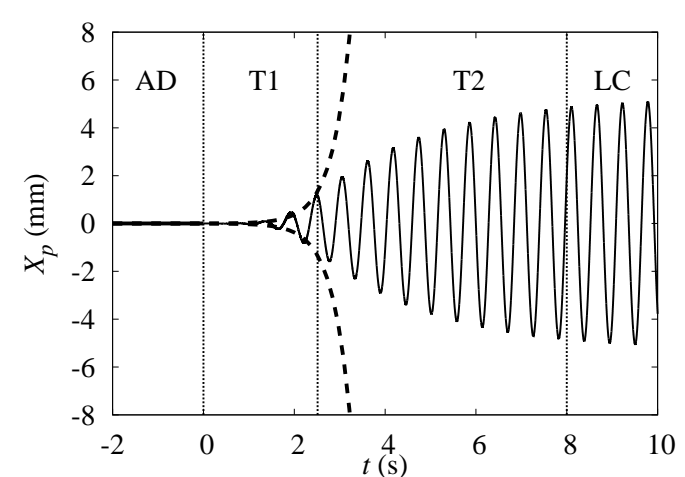

(a)

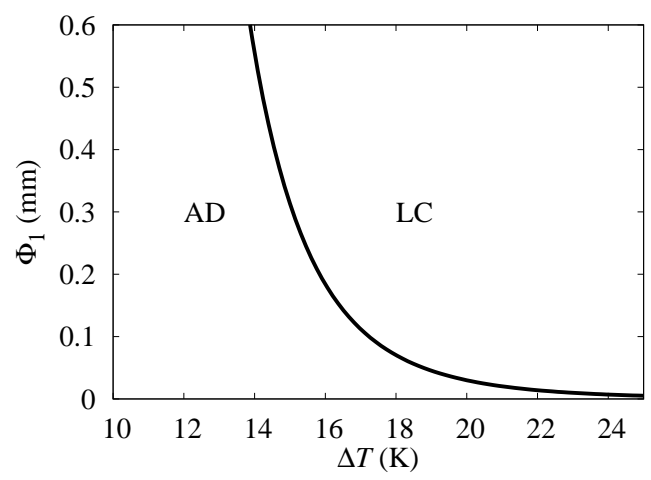

(c)

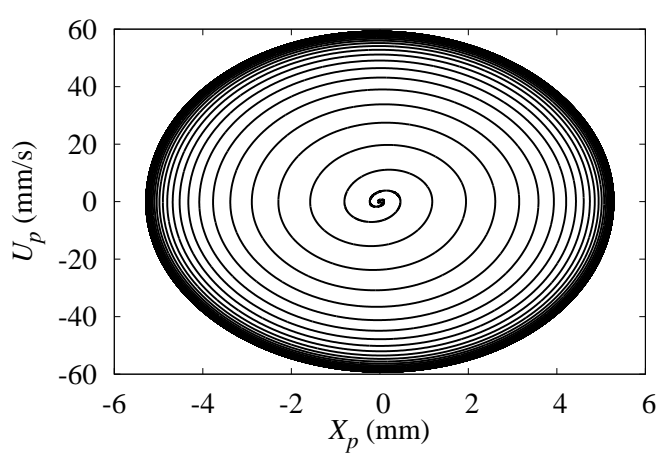

(b)

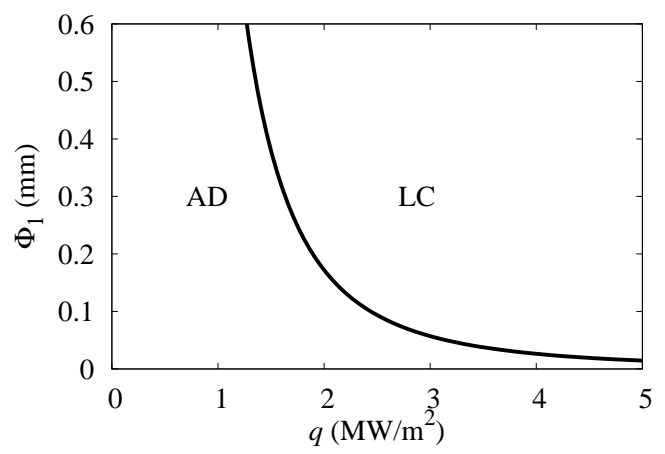

(d)

FIG. 11. Phase diagram in our dynamical systems. (a) Transition from AD to LC; (b) Limit cycle; (c) Two parameter ( $\Phi_{1}$ vs $\Delta T$ ) phase diagram denoting the transition between AD to LC for Model A; (d) Two parameter ( $\Phi_{1}$ vs $q$ ) phase diagram denoting the transition between AD to LC for Model B.

the peak frequency $f_{p}$ is $1.64 \mathrm{~Hz}$ for the transition and steady states. Thus, we find that $f_{p}$ obtained by FFT agrees with $f_{s}=1.75 \mathrm{~Hz}$ (which is discussed in Sec. III) within the frequency accuracy $\left(\Delta f=\frac{1}{n \Delta t}=0.234 \mathrm{~Hz}\right)$ of the FFT, where $\Delta t=0.008333 \mathrm{~s}$ is the time interval of the date. In other words, from Fig. 10, we confirm that the oscillation of the self-propelled swing motion is the simple harmonic oscillation, as discussed in Sec. III. Note that it is well known that the width of the peak in Fig. 10 is caused by the finite sampling within a finite time. 


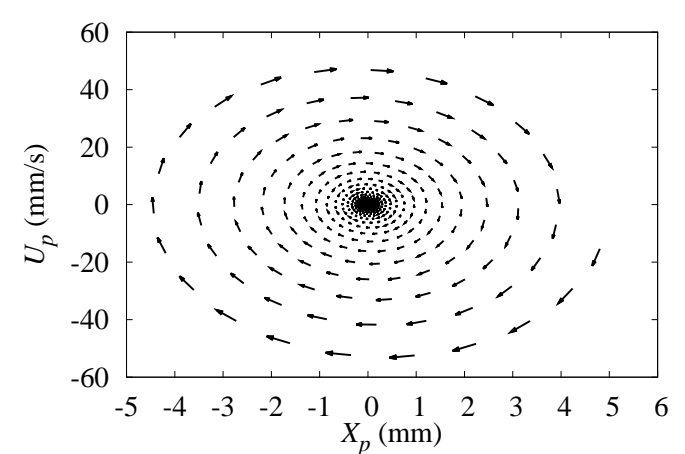

(a)

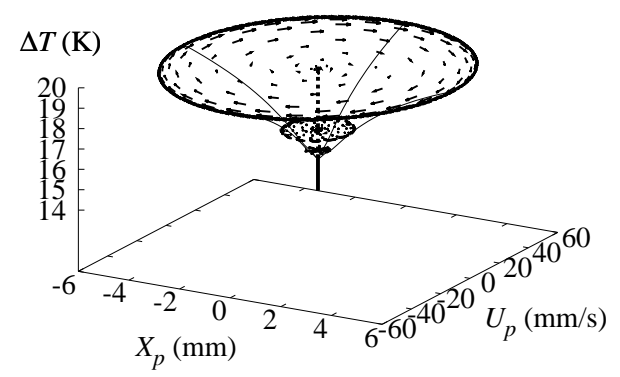

(c)

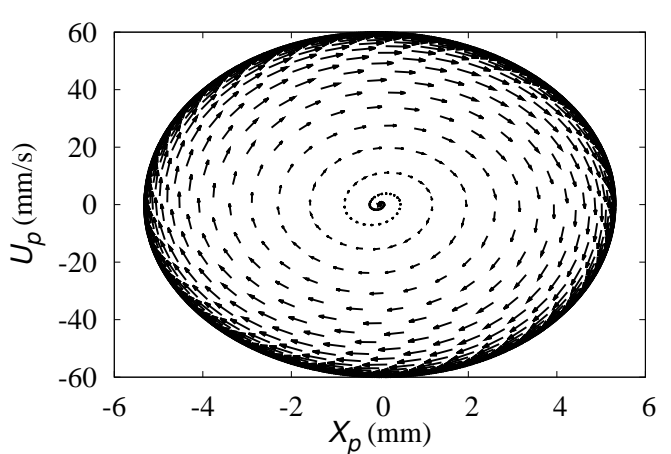

(b)

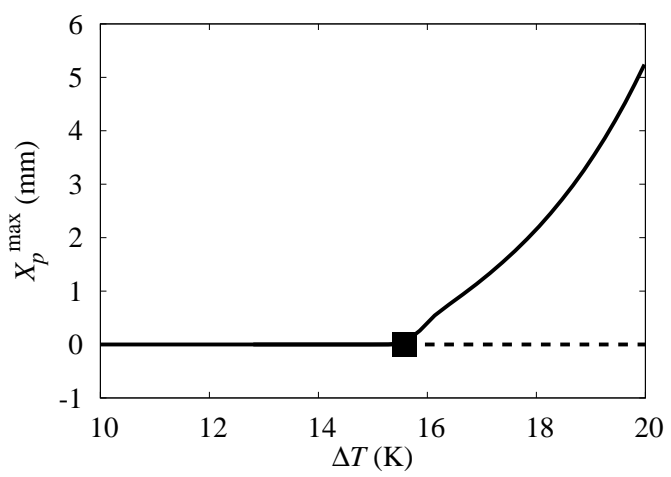

(d)

FIG. 12. Bifurcation structures in our dynamical systems. (a) "Flow field" of our dynamical systems at $\Delta T=0 \mathrm{~K}$; (b) "Flow field" of our dynamical systems at $\Delta T=20 \mathrm{~K}$; (c) 3D bifurcation diagram at $\Phi_{1}=0.281 \mathrm{~mm}$; (d) Schematic bifurcation diagram $\Phi_{1}=0.281 \mathrm{~mm}$. In (d), the solid and broken lines show stable and unstable solutions, respectively, while the closed square shows the Hopf-type bifurcation point.

\section{About bifurcation structures}

It is important to clarify bifurcation structures (changes in the qualitative nature of the dynamics) in dynamical systems [23-25]. Figure 11 shows a phase diagram in our dynamical systems. Specifically, Fig. 11(a) shows the transition from an amplitude death (AD) state to a limit cycle (LC) state through two transient states (T1 and T2). Here, T1 is a transient state that can be approximated by the linearized equation Eq. (8) while T2 is the other transient state. Further, the calculation corresponds to Fig. 8(b), while AD is the term introduced by Koseska et al, [23] to explain structurally different oscillation quenching types. Although the situation is a little different, we consider that similar transition occurs in our 
dynamical systems. That is, by applying $V_{0}$ at $t=0 \mathrm{~s}, \Delta T$ becomes nonzero values and thus the transition from a stable $\mathrm{AD}$ to $\mathrm{T} 1$ occurs; then, the transition from $\mathrm{T} 1$ to $\mathrm{T} 2$ occurs (i.e., suppression of the oscillation starts) at $t \simeq 2.5 \mathrm{~s}$ and reaches a stable limit cycle at $t \geq 8 \mathrm{~s}$, as shown in Fig. 11(a). In Fig. 11(a), broken lines show the envelop of the initial linear solution that represents the T1 state, which corresponds to Eq. (8). Thus, we find that the nature of dynamics changes at $t \simeq 2.5 \mathrm{~s}$ in Fig. 11(a). Furthermore, Fig. 11(b) shows a trajectory of $\left(X_{p}, U_{p}\right)$ at $t=0$ to $40 \mathrm{~s}$, which corresponds to Fig. 11(a). As shown in Fig. 11(b), we find that a limit cycle is formed in our dynamical systems.

Figure 11(c) shows the two parameter $\left(\Phi_{1}\right.$ vs $\left.\Delta T\right)$ phase diagram denoting the transition between AD to LC for Model A, while Fig. 11(d) shows the two parameter ( $\Phi_{1}$ vs q) phase diagram denoting the transition between $\mathrm{AD}$ to $\mathrm{LC}$ for Model B. Here, $\Phi_{1}$ is defined as

$$
\Phi_{1} \equiv \frac{A}{A_{v}} \frac{L-0.5 L_{w}}{L} \phi_{1}
$$

and it describes the transition of our dynamical systems along with $\Delta T$. That is, from Eq. (9), we obtain the transition condition as follows:

$$
\Phi_{1}(\Delta T)=\frac{24 \mu u_{c}}{\Delta P(\Delta T) R_{b}(\Delta T)} .
$$

From Figs. 11(c) and 11(d), we find that larger $\Delta T$ and $q$ values are required for the transition from $\mathrm{AD}$ to LC, as $\Phi_{1}$ decreases. Note that physically Figs. 11(c) and 11(d) provide design criteria for our device. For example, we obtain $\Phi_{1}=0.281 \mathrm{~mm}$ for the conditions of Fig. 11(a) and thus the $\Delta T$ and $q$ values for the transition are approximately $15 \mathrm{~K}$ and $1.8 \mathrm{MW} / \mathrm{m}^{2}$, respectively.

Figure 12 shows bifurcation structures in our dynamical systems. Specifically, Fig. 12(a) shows the "flow field" of our dynamical systems at $\Delta T=20 \mathrm{~K}$, while Fig. 12(b) shows the "flow field" of our dynamical systems at $\Delta T=0 \mathrm{~K}$. Here, the "flow field" is the specific technical term in dynamical systems and it is defined as the vector field $\left(V_{x}, V_{y}\right)=\left(\dot{X}_{p}, \dot{U}_{p}\right)$. As shown in Figs. 12(a) and 12(b), the stable equilibrium point $\boldsymbol{O}\left[=\left(X_{p}, U_{p}\right)=(0,0)\right]$ becomes unstable by changing the parameter $\Delta T$ from 0 to $20 \mathrm{~K}$. As a result, a stable LC state is generated. Since the bifurcation that accompanies the destabilization of a stable equilibrium point and the generation of LC is generally called Hopf bifurcation, the observed phenomenon is regarded as Hopf bifurcation. Figure 12(c) shows a 3D bifurcation diagram at $\Phi_{1}=0.281 \mathrm{~mm}$, whereas Fig. $12(\mathrm{~d})$ shows a schematic bifurcation diagram $\Phi_{1}=0.281$ 
mm. As shown in Figs. 12(a) and 12(b), we find that our dynamical systems have the bifurcation structure similar to the well-known typical Hopf bifurcation structure. Note that in Fig. 12(d), the solid and broken lines show stable and unstable solutions, respectively, while the closed square shows the Hopf-type bifurcation point.

\section{E. Originality of our device}

Originality of our study is very high because (1) there has been no heat engine consisting only of nichrome wires so far. Who thinks an engine can be made with just a nichrome wire? Thus, our device is important as the seed of the new technology for the ultimate small heat engine in the future. (2) Although there are many works on self-propulsion [26], there is no work for the self-propulsion using the self increasing phenomenon of the temperature difference between the forward and rear surfaces. In particular, no one consider that the symmetrical heating element moves in a specific direction by itself faster and faster until the driven force due to the bubble balances the viscous force. However, our experimental and theoretical results tell us that such phenomenon will intrinsically occur in the transition boiling region if the condition of Eq. (9) is satisfied. Thus, our device is innovative and it is important as a micro-heat engine with strong rotational power that cannot be imitated by other methods. (3) There is no miniaturizable device that runs at speed of $6 \mathrm{~cm} / \mathrm{s}$ or more [e.g., 14 cm/s in Fig. 9(b)] in water and can be used for the microfluidic applications. For example, the velocity of the promising movable device using induced charge electro-osmosis with a Janus structure is $\sim 1 \mathrm{~mm} / \mathrm{s}$ at most [27]. Furthermore, although Linke et al.'s device [1] can propel a droplet with $\sim 5 \mathrm{~cm} / \mathrm{s}$ on a hot ratchet surface, application to the movable devices that can be driven in water can not be expected; i.e., it will not work in a microfluidic channel. Thus, our device is promising as an innovative high-speed microfluidic device that can be driven in water, since our device can intrinsically be driven in water all of the part, although we demonstrate our device with a pendulum immersed partially in water as the first step. In the future, we will demonstrate such innovative microfluidic devices. 


\section{CONCLUSION}

In conclusion, we have reported a strong self-propelled swing motion of pendulums having a hot tip immersed in water. Further, we have proposed a model explaining that the strong swing motion occurs due to the growing asymmetrical instability of heat transfer from the hot tip to the water.

\section{Appendix A: Detail explanations on $R_{a}$ and $R_{b}$}

\section{Force acting on the surface of the heater in the numerate regime}

The number of bubbles that generate on the heater of area $\frac{A}{2}$ during $1 \mathrm{~s}$ is written as

$$
N_{a}\left(\Delta T^{*}\right)=N_{n} \frac{A}{2} f_{g}
$$

where $\Delta T^{*}$ is the superheat for each surface, $N_{n}\left(\Delta T^{*}\right)$ is the number density of the active nucleation site, and $f_{g}$ is the bubble generation frequency from the active sites [28]. Thus, we can write the average force that the seed of the bubbles provides to the heater at the one side as

$$
f_{b s}\left(\Delta T^{*}\right) \simeq N_{a} \frac{\pi D_{d}^{2}}{4} \Delta P \Delta t_{c} \simeq \frac{\pi D_{d}^{2} f_{g} \Delta t_{c}}{4} \frac{A \Delta P}{2} N_{n}
$$

where $D_{d}$ is the average departure diameter of the bubbles, $\Delta t_{c}$ is the average effective deforming time, and $f_{g} \Delta t_{c}(\leq 1)$ is the effective bubble working ratio. As for $N_{n}$, several models exist [28]. For instance, Yang and Kim [29] proposed that

$$
N_{n}\left(\Delta T^{*}\right)=N_{0} \mathrm{e}^{-\frac{K}{\Delta T^{*}}}
$$

where $N_{0}$ and $K$ are constant values. Thus, from Eqs. (A6) and (A7), we obtain

$$
f_{b s}\left(\Delta T^{*}\right) \simeq \frac{\pi D_{d}^{2} N_{0} f_{g} \Delta t_{c}}{4} \mathrm{e}^{-\frac{K}{\Delta T^{*}}} \frac{A \Delta P}{2}=\frac{A \Delta P}{2} R_{b}^{\prime}
$$

Here,

$$
R_{b}^{\prime}\left(\Delta T^{*}\right) \equiv \frac{\pi D_{d}^{2} N_{0} f_{g} \Delta t_{c}}{4} \mathrm{e}^{-\frac{K}{\Delta T^{*}}}
$$

is the effective surface bubble coverage rate for the specific nucleate-boiling model and it consists of the effective bubble working ratio part $\left(f_{g} \Delta t_{c}\right)$ and the surface bubble coverage rate part at the initial stage of the bubble generation $\left(\frac{\pi D_{d}^{2} N_{0}}{4} \mathrm{e}^{-\frac{K}{\Delta T^{*}}}\right)$. From the above 
argument, the net force acting on the heater is written as

$$
f_{b}^{\text {Model } C}=f_{b s}\left(\Delta T_{r}^{*}\right)-f_{b s}\left(\Delta T_{f}^{*}\right)
$$

where the subscript "f" and "r" denote the surfaces of the cylindrical heater at the front and rear sides, respectively. Note that the seeds of the bubbles are generated with the departure diameter instantaneously and leaving the heaters with the lift-off diameter. During this process, the bubbles push water and the heater. Although those forces of bubbles are intrinsically discontinuous in time and have different values depending on their size at different positions in space, we can consider that bubbles continuously exert a force on the heater by considering the average working area $A_{d}=\frac{\pi D_{d}^{2}}{4}$ in space and the effective bubble working ratio $r_{t}=f_{g} \Delta t_{c}$ in time. In other words, by using $A_{d}$ and $r_{t}$ within the framework of ordinary statistical methods, we approximate a discontinuous force exerted on a heater due to the many bubble generation events as the continuous average force due to bubbles in Eq. (A2).

\section{Model for the surface temperature of the heater}

It is known that temperature $T$ and heat transfer coefficient $\alpha$ around a cylinder vary with the position in a flow [30]. In other words, there is a tendency that $\alpha$ at the front side is larger than that at the rear side because the flow velocity at the rear side is slower than that at the front side. Although detail information is not known, we can assume that

$$
\begin{array}{r}
\Delta T_{f}^{*} \simeq \Delta T-\delta T\left(U_{p}\right), \\
\Delta T_{r}^{*} \simeq \Delta T,
\end{array}
$$

as the first step. Here, $\Delta T_{r}^{*}-\Delta T_{f}^{*}=\delta T\left(U_{p}\right)$ and $\delta T$ approaches $\Delta T$ at $U_{p}=\infty$, while $\delta T$ is mathematically proportional to $U_{p}$ at $U_{p} \ll u_{c}^{\prime}$ by the approximation using a Taylor series expansion since $\delta T$ is a function of $U_{p}$ for the constant $\Delta T$. Here, $u_{c}^{\prime}$ is the represented velocity. Thus, we assume that

$$
\delta T \simeq\left(1-e^{-\frac{U_{p}}{u_{c}^{p}}}\right) \Delta T=R_{a}^{\prime}\left(U_{p}\right) \Delta T
$$

where $R_{a}^{\prime}\left(U_{p}\right)=1-e^{-\frac{U_{p}}{u_{c}^{\prime}}}$. Therefore, by using Eqs. (A6) to (A9), we obtain $f_{b}^{\text {Model } C}$ principally. However, $f_{b}^{\text {Model } C}$ approaches $f_{b s}(\Delta T)$ at $U_{p}=\infty$, while $f_{b}^{\text {Model } C}$ is mathematically 
proportional to $U_{p}$ at $U_{p} \ll u_{c}$ by the approximation using a Taylor series expansion since $f_{b}^{\text {Model } C}$ is a function of $U_{p}$ for the constant $\Delta T$. Thus, by assuming separation of variables, we can approximate that

$$
f_{b}^{\text {Model } C} \simeq\left(1-e^{-\frac{U_{p}}{u_{c}}}\right) f_{b s}(\Delta T)=R_{a}\left(U_{p}\right) f_{b s}(\Delta T)
$$

since $R_{a} \simeq \frac{U_{p}}{u_{c}}$ at $U_{p} \ll u_{c}$ and $R_{a} \simeq 1$ at $U_{p} \ll \infty$. Therefore, by substituting Eq. (A5) into (A10), we obtain

$$
f_{b}^{\text {Model } C} \simeq R_{a}\left(U_{p}\right) R_{b}^{\prime}(\Delta T) \frac{A \Delta P(\Delta T)}{2}
$$

Note that we propose that the expression $R_{a}\left(U_{p}\right)=1-e^{-\frac{U_{p}}{u_{c}}}$ as the continuous function that combines the function $R_{a} \simeq \frac{U_{p}}{u_{c}}$ at $U_{p} \ll u_{c}$ with the function $R_{a} \simeq 1$ at $U_{p} \ll \infty$ under the assumption that the beginning and end of acceleration are more important than the intermediate process for our self-swing motions. This is the origin of $R_{a}$. Furthermore, the choice of $\Delta P(\Delta T)$ in Eqs. (A2), (A4), and (A11) indicates that what sets the pendulum in motion is fundamentally the vapor pressure difference in the initial superheat layer that is the seed of the bubbles between the front and the rear, due to a temperature difference $\delta T=R_{a}^{\prime}\left(U_{p}\right) \Delta T$.

\section{Needs for the formulation that covers the nucleate- and film-boiling regimes}

$R_{b}^{\prime}$ in Eq. (A5) is the origin of $R_{b}$ and it shows a physical meaning of $R_{b}$ in the nucleateboiling regime. Furthermore, we can calculate by providing appropriate parameters in Eq. (A5); e.g., as the first step, we may use

$$
\begin{aligned}
D_{d} \sim 0.0208 \theta_{w} \sqrt{\frac{\sigma}{g \Delta \rho}}, \\
\Delta t_{c} \sim \frac{1}{2 f_{g}}, \\
f_{g} \Delta t_{c} \sim \frac{1}{2},
\end{aligned}
$$

on the basis of the knowledge of the bubble dynamics [31], where $\theta_{w}$ is a contact angle of water measured in degrees, $\sigma$ is a surface tension of water, $g$ is the gravitational acceleration, and $\Delta \rho=\rho_{l}-\rho_{v}$ is a density difference between liquid and gas phases. Note that Eq. (A12) is well known as Fritz equation and one can find more complex formulations for the parameters in many papers; e.g., you can see the review by Dhir [32]. However, in the nucleate boiling 
region, the effect of individual surfaces is strong. Thus, there is no definitive model that can be accepted by every researchers, although every models are valid to some extent. Therefore, details of the formulation of $R_{b}^{\prime}$ might not be so important. In fact, by using Kocamustafagullari and Ishii's model [28, 33] instead of Yang and Kim's model [29], we can rewrite Eqs. (A3) and (A5) as

$$
N_{n}(\Delta T) \simeq N_{0} \Delta T^{4.4}
$$

and

$$
R_{b}^{\prime}(\Delta T) \simeq \frac{\pi D_{d}^{2} N_{0} f_{g} \Delta t_{c}}{4} \Delta T^{4.4}
$$

In particular, for our self-swing problem, we consider that the most important fact is that $R_{b}^{\prime}$ is proportional to the $n^{\prime}$ power of $\Delta T$, where $n^{\prime}=4.4$ but it is replaced by the more suitable value $n=8$ for $R_{b}$. Moreover, although $R_{b}^{\prime}$ is useful in the nucleate-boiling region, it is not useful in the film-boiling region in spite of the fact that our self-propelled swing motions occur in the nucleate- to film-boiling regions (in particular, in the transition boiling region). Therefore, we need the other formulation that covers the nucleate- and film-boiling regions for the effective coverage rate.

\section{Concepts of $R_{b}$}

Our self-swing phenomenon occurs at $q \sim 1.5$ to $6 \mathrm{MW} / \mathrm{m}^{2}$, which is corresponding to the transition boiling region. In the transition- to film-boiling regions, the effects of surface individuality are thought to be lost because of the overlap of the bubbles and the initiation of the spontaneous nucleation [33]. Thus, the effective coverage rate $R_{b}(\Delta T)$ that considers to be an effective utilization rate for the force $\frac{A}{2} \Delta P$ becomes more useful than $R_{b}^{\prime}(\Delta T)$ that considers the details mechanism in particular for $N_{n}$. Furthermore, although one may consider that $R_{b} \sim 1$ in the transition- and film-boiling regions, it is not correct, since it is generally accepted that the boiling liquid does contact the solid heat transfer surface during transition boiling; e.g. Dhuga and Winterton [22] shows that the fraction of wetted area starts to decrease at $\Delta T \sim 20 \mathrm{~K}$ and drops rapidly at $\Delta T \sim 60 \mathrm{~K}$ from the impedance measurement of water. Thus, we need to consider which $\Delta T$ captures the characteristics of $R_{b}(\Delta T)$ for $\frac{A}{2} \Delta P$. Specifically, we consider the Leidenfrost temperature $\Delta T_{c}=60 \mathrm{~K}$ and the fact that the initial pressure of the superheat layer is $\sim 4.5 \mathrm{MPa}$ at $\Delta T \sim 170 \mathrm{~K}$ [19], as mentioned in Sec. IV. In other words, we consider that $R_{b}$ should increase rapidly 
at $\Delta T_{c} \sim 60 \mathrm{~K}$ and $R_{b} \Delta P \sim 4.5$ at $\Delta T \sim 170 \mathrm{~K}$. In addition, since $R_{b}^{\prime} \propto \Delta T^{n^{\prime}}$ in Eq. (A16), we consider that $R_{b} \propto \Delta T^{n}$ at $\Delta T \ll \Delta T_{c}$. Therefore, we propose the expression $R_{b}=\left(1-\mathrm{e}^{-\frac{\Delta T}{\Delta T_{c}}}\right)^{8}$, since it satisfies the above conditions. These are the concepts of $R_{b}$. Note that as explained in this session, $R_{a}$ and $R_{b}$ consist of very few simple concepts but they depict our experiments clearly, since the effects of surface individuality disappear in the transition boiling region of $q \sim 1.5$ to $6 \mathrm{MW} / \mathrm{m}^{2}$.

[1] H. Linke, B. J. Alemán, L. D. Melling, M. J. Taormina, M. J. Francis, C. C. Dow-Hygelund, V. Narayanan, R. P. Taylor, and A. Stout, Phys. Rev. Lett. 96, 154502 (2006).

[2] D. Quéré, Annual Review of Fluid Mechanics 45, 197 (2013).

[3] A.-L. Biance, C. Clanet, and D. Qur, Physics of Fluids 15, 1632 (2003).

[4] J. T. Ok, E. Lopez-Oña, D. E. Nikitopoulos, H. Wong, and S. Park, Microfluidics and Nanofluidics , 1045 (2011).

[5] Q. Li, Q. J. Kang, M. M. Francois, and A. J. Hu, Soft Matter 12, 302 (2016).

[6] A. Würger, Phys. Rev. Lett. 107, 164502 (2011).

[7] A. Hashmi, Y. Xu, B. Coder, P. A. Osborne, J. Spafford, G. E. Michael, G. Yu, and J. Xu, Scientific Reports 2, 797 (2012).

[8] G. G. Wells, R. Ledesma-Aguilar, G. McHale, and K. Sefiane, Nature Communications 6, 639 (2015).

[9] M. Shi, X. Ji, S. Feng, Q. Yang, T. J. Lu, and F. Xu, Scientific Reports 6, 28574 (2016).

[10] H. Sugioka and S. Segawa, AIP Advances 8, 115209 (2018).

[11] H. Xu, A. Thissandier, R. Zhao, P. Tao, C. Song, J. Wu, W. Shang, and T. Deng, Applied Physics Letters 114, 113703 (2019).

[12] C. Luo, M. Mrinal, and X. Wang, Scientific Reports 7, 12018 (2017).

[13] J. T. Pham, M. Paven, S. Wooh, T. Kajiya, H.-J. Butt, and D. Vollmer, Nature Communications 8, 905 (2017).

[14] A. Bouillant, T. Mouterde, P. Bourrianne, A. Lagarde, C. Clanet, and D. Quéré, Nature Physics 10, 1038 (2018).

[15] K. Nishikawa and Y. Fujita (Academic Press, Inc., 1990) p. 1. 
[16] JSME, JSME Mechanical Engineers' Handbook: Fundamentals: a4: Fluids Engineering (The Japan Society of Mechanical Engineers, 2006).

[17] G. G. Stokes, Trans. Cambridge. Philos. Soc. 9, 8 (1851).

[18] H. Sugioka, S. Segawa, and M. Kubota, Journal of Applied Physics 125, 134502 (2019).

[19] A. Asai, T. Hara, and I. Endo, Japanese Journal of Applied Physics 26, 1794 (1987).

[20] J. M. den Toonder and P. R. Onck, Trends in Biotechnology 31, 85 (2013).

[21] S. Hanasoge, P. J. Hesketh, and A. Alexeev, Microsystems \& Nanoengineering 4, 11 (2018).

[22] D. Dhuga and R. Winterton, International Journal of Heat and Mass Transfer 28, 1869 (1985).

[23] A. Koseska, E. Volkov, and J. Kurths, Phys. Rev. Lett. 111, 024103 (2013).

[24] R. Kapral and P. Mandel, Phys. Rev. A 32, 1076 (1985).

[25] L. Glass, M. R. Guevara, J. Belair, and A. Shrier, Phys. Rev. A 29, 1348 (1984).

[26] J. L. Moran and J. D. Posner, Annual Review of Fluid Mechanics 49, 511 (2017).

[27] S. Gangwal, O. J. Cayre, M. Z. Bazant, and O. Velev, Phys. Rev. Lett. 100, 058302 (2008).

[28] T. Hibiki and M. Ishii, International Journal of Heat and Mass Transfer 46, 2587 (2003).

[29] S. Yang and R. Kim, International Journal of Heat and Mass Transfer 31, 1127 (1988).

[30] J. Giddings, P. Shinude, and S. Semenov, J. Colloid and interface science 176, 454 (1995).

[31] C. Yang, Y. Wu, X. Yuan, and C. Ma, International Journal of Heat and Mass Transfer 43, $203(2000)$.

[32] V. Dhir, International Journal of Heat and Fluid Flow 12, 290 (1991).

[33] G. Kocamustafaogullari and M. Ishii, International Journal of Heat and Mass Transfer 26, $1377(1983)$. 